\title{
NETS: Network estimation for time series
}

\author{
Matteo Barigozzi ${ }^{1} \mid$ Christian Brownlees ${ }^{2}$
}

\author{
${ }^{1}$ Department of Statistics, London School \\ of Economics and Political Science, \\ London, UK \\ ${ }^{2}$ Department of Economics and Business, \\ Universitat Pompeu Fabra and Barcelona \\ GSE, Barcelona, Spain

\section{Correspondence} \\ Matteo Barigozzi, Department of \\ Statistics, London School of Economics \\ and Political Science, Houghton Street, \\ London WC2A 2AE, UK. \\ Email: m.barigozzi@lse.ac.uk
}

\begin{abstract}
Summary
We model a large panel of time series as a vector autoregression where the autoregressive matrices and the inverse covariance matrix of the system innovations are assumed to be sparse. The system has a network representation in terms of a directed graph representing predictive Granger relations and an undirected graph representing contemporaneous partial correlations. A LASSO algorithm called NETS is introduced to estimate the model. We apply the methodology to analyze a panel of volatility measures of 90 blue chips. The model captures an important fraction of total variability, on top of what is explained by volatility factors, and improves out-of-sample forecasting.
\end{abstract}

\section{1 | INTRODUCTION}

Over the last years, network analysis has become an active topic of research in time series econometrics, with numerous applications in macroeconomics and finance. Examples of contributions in the literature include, among others, Billio, Getmansky, Lo, and Pellizzon (2012), Diebold and Yllmaz (2014, 2015), Hautsch, Schaumburg, and Schienle (2015), and Härdle, Wang, and Yu (2016). In a nutshell, network analysis is concerned with representing the interconnections of a large panel as a graph: the vertices of the graph represent the variables in the panel, and the presence of an edge between two vertices denotes the presence of some appropriate measure of dependence between the two variables. From an economic perspective, the interest on networks has been boosted by the research of Acemoglu, Carvalho, Ozdaglar, and Tahbaz-Salehi (2012), for example, which shows that individual entities can have a non-negligible effect on the aggregate behavior of the economy when the system has a high degree of interconnectedness.

In this paper we propose network methodology for large panels of time series. We model the panel as a vector autoregression (VAR). We work under the assumption that the VAR is sparse, in the sense that the autoregressive matrices and the inverse covariance matrix of the system innovations are assumed to be sparse. Note that the notion of sparsity used in this work is different from that used in other papers such as Davis, Zang, and Zheng (2016), Kock and Callot (2015), and Medeiros and Mendes (2016), where sparsity assumptions are formulated for the autoregressive matrices only. Sparsity of the autoregressive matrices implies sparsity of the multivariate Granger causality structure of the system, whereas sparsity of the inverse covariance matrix implies sparsity of the partial correlation structure (Dempster, 1972; Lauritzen, 1996).

Several network representations can be associated with a VAR system (Dahlhaus, 2000; Diebold \& Y1lmaz, 2014; Eichler, 2007). In this work we focus on two representations that are natural for the sparse VAR we introduce in this work. The first network representation consists of representing the system as a mixed graph containing both directed and undirected edges: directed edges denote Granger causality linkages among time series, while undirected edges represent contemporaneous partial correlation linkages. The second network representation we introduce is an undirected graph, where edges denote long-run partial correlation linkages among time series. Long-run partial correlation is a partial correlation measure constructed on the basis of the long-run covariance matrix of the VAR. It synthesizes simultaneously lead/lag and contemporaneous dependence among time series and is a natural generalization for dependent data of the standard partial correlation model used in the statistics graphical literature. 
In order to estimate large sparse VARs we introduce a novel LASSO-based algorithm. The highlight of the procedure is that it simultaneously estimates the autoregressive matrices as well as the entries of the concentration matrix, avoiding splitting up the estimation of the model parameters into two steps. The large-sample properties of the proposed estimator are analyzed and we establish conditions for consistent selection and estimation of the VAR parameters. The theory is derived in a high-dimensional setting, allowing the number of series in the system to increase with the sample size. Specifically, the number of series is allowed to be $O\left(T^{\zeta}\right)$ for $\zeta>0$, where $T$ denotes the sample size of the panel.

The network methodology we introduce in this work has highlights in terms of interpretation and estimation. Understanding and synthesizing the interdependence structure of a large multivariate system can be a daunting task. The network representation of the panel provides a parsimonious synthesis of the data that can bring useful insights to their underlying structure. From an estimation perspective, carrying out inference on the VAR parameters can be challenging when the number of time series is large. The regularized estimation approach based on LASSO put forward in this work can lead to substantial gains in terms of estimation precision and, ultimately, forecasting. The gains of the methodology rely on sparsity assumptions of the underlying system. It is important to emphasize that these assumptions may not always be appropriate. In practice, users ought to check whether these are plausible for the data at hand and eventually, when possible, appropriately transform their data.

A natural application of network analysis techniques is the study of interdependence in panels of volatility measures. Detecting the interconnectedness structure of volatility panels is of interest to understand and monitor the risk transmission channels of these systems. See, for instance, the research of Diebold and Yllmaz (2014) on risk transmission in the 2007-2009 great financial crisis or Engle, Gallo, and Velucchi (2012) in the 1997-1998 Asian financial crisis. We use the methodology derived in this work to analyze a panel of volatility measures for 90 US blue chips across different industry groups from January 2, 2004, to December 31, 2015. An important feature of our application is that we study interconnectedness conditional on a market-wide and sector-specific volatility factors. We show that after conditioning on the factors the sparse VAR captures approximately $10 \%$ of the overall variability. The estimated networks connect the vast majority of the series in the panel and the interdependence is positive in the vast majority of cases. Results show that the financial sector is the most interconnected industry in this sample period. In particular, large financial institutions such as AIG, Bank of America, and Citigroup are some of the most interconnected entities in the panel. An out-of-sample forecasting exercise is used to validate the methodology proposed in our work and shows that the sparse VAR model improves predictive ability over a number of benchmarks.

Our work relates to different strands of literature. First, it is related to the econometric literature on networks, which includes research by Billio et al. (2012), Diebold and Yllmaz (2014, 2015), Hautsch et al. (2015), Anufriev and Panchenko (2015), Härdle et al. (2016), and Hagströmer and Menkveld (2016). Early influential work on networks and panels of time series includes Mantegna (1999). This paper is also related to the literature on the estimation of sparse VARs (see Basu \& Michailidis, 2015; Davis et al., 2016; Kock \& Callot, 2015; Medeiros \& Mendes, 2016; Song \& Bickel, 2011). Our contribution also relates to the statistical literature on large-dimensional network estimation based on LASSO techniques. Contributions in this area include, among others, Meinshausen and Bühlmann (2006), Friedman, Hastie, and Tibshirani (2008), and Peng, Wang, Zhou, and Zhu (2009).

The paper is structured as follows. Section 2 introduces the model, the network definitions, and the estimation strategy. Section 3 contains a simulation study that analyzes the finite-sample properties of the procedure. Section 4 contains the empirical application. Concluding remarks follow in Section 5. The proofs of all our results may be found in a Supporting Information Appendix, available online.

\section{2 | METHODOLOGY}

\section{1 | Model}

We consider a zero-mean stationary $n$-dimensional multivariate time series $\mathbf{y}_{t}=\left(y_{1 t}, \ldots, y_{n t}\right)^{\prime}$ generated by a $p$ th-order VAR:

$$
\mathbf{y}_{t}=\sum_{k=1}^{p} \mathbf{A}_{k} \mathbf{y}_{t-k}+\boldsymbol{\epsilon}_{t}, \boldsymbol{\epsilon}_{t} \sim \text { i.i.d. }\left(\mathbf{0}, \mathbf{C}^{-1}\right),
$$

where $\mathbf{A}_{k}$ and $\mathbf{C}$ are $n \times n$ matrices. Throughout, the VAR is assumed to be stable and $\mathbf{C}$ to be positive definite. Note that for convenience the distribution of the innovation terms is parametrized with the inverse covariance matrix $\mathbf{C}$, also known as concentration matrix, rather than the covariance. The $(i, j)$ th entries of the matrices $\mathbf{A}_{k}$ and $\mathbf{C}$ are denoted, respectively, by $a_{k i j}$ and $c_{i j}$. 
In this work we focus on the analysis of sparse VAR systems, in the sense that the autoregressive matrices $\mathbf{A}_{k}$ and the concentration matrix $\mathbf{C}$ are assumed to be sparse matrices. More specific notions of sparsity are spelled out in Section 2.4, where precise assumptions are required by the estimation theory to establish the results of interest. In general, the sparsity assumption can be interpreted as a sparsity assumption on the lead/lag and contemporaneous dependence structure of the system.

The standard notion of dynamic interdependence used for time series is Granger causality. In this work we rely on a multivariate version of this concept. Formally, we say that $y_{j t}$ does not Granger cause $y_{i t}$ if adding $y_{j t}$ as predictor does not improve the mean square forecast error of $y_{i t+k}$ for any $k>0$; that is:

$$
\mathrm{E}\left[\left(y_{i t+k}-\mathrm{E}\left(y_{i t+k} \mid\left\{y_{1 t} \cdots y_{n t}\right\}\right)\right)^{2}\right]=\mathrm{E}\left[\left(y_{i t+k}-\mathrm{E}\left(y_{i t+k} \mid\left\{y_{1 t} \cdot y_{n t}\right\} \backslash y_{j t}\right)\right)^{2}\right] .
$$

We can immediately see that the Granger causality structure of the model is encoded in the sparsity structure of the autoregressive matrices $\mathbf{A}_{k}$. We have indeed that if $a_{k i j}=0$, for all $k$, then $y_{j t}$ does not Granger cause $y_{i t}$.

The classical measure of contemporaneous dependence used in the network literature is partial correlation. In this paper we consider partial correlation between two series conditional on the past realizations of the panel and contemporaneous realizations of the remaining series. This is encoded in the partial correlation between VAR innovations, which is denoted by

$$
\rho^{i j}=\operatorname{corr}\left(\epsilon_{i t}, \epsilon_{j t} \mid\left\{\epsilon_{k t}: k \neq i, j\right\}\right) .
$$

It is well known that partial correlations are related to the entries $c_{i j}$ of the concentration matrix $\mathbf{C}$ by means of the relation (Dempster, 1972)

$$
\rho^{i j}=-\frac{c_{i j}}{\sqrt{c_{i i} c_{j j}}}
$$

Thus the contemporaneous dependence sparsity structure is embedded in the sparsity structure of the concentration matrix $\mathbf{C}$. Indeed, if $c_{i j}=0$, then series $i$ and $j$ are contemporaneously uncorrelated conditional on all other series in the system.

Networks are a useful tool to represent the interdependence structure of the time series in the panel $\mathbf{y}_{t}$. A network is defined as a graph $\mathcal{N}=(\mathcal{V}, \mathcal{E})$, where $\mathcal{V}$ is the set of vertices and $\mathcal{E}$ is the set of edges. The set of vertices $\mathcal{V}$ is $\{1, \ldots, n\}$, where each element corresponds to a component of $\mathbf{y}_{t}$, while the set of edges $\mathcal{E}$ is a subset of $\mathcal{V} \times \mathcal{V}$ such that the pair $(i, j)$ is in $\mathcal{E}$ if and only if the components $i$ and $j$ are linked by an edge. Several network representations can be associated with a VAR system. In this work we focus on two representations that are natural for the sparse VAR we introduce in this work.

A natural representation of the sparse VAR model is based on the union of two graphs: the first graph contains directed edges denoting Granger causality linkages, whereas the second graph contains undirected edges representing contemporaneous partial correlation linkages. We label the two networks respectively as the Granger and contemporaneous networks. The Granger network is defined as a directed network $\mathcal{N}_{G}=\left(\mathcal{V}, \mathcal{E}_{G}\right)$, where the presence of an edge from $i$ to $j$ denotes that $i$ Granger causes $j$ in the sense of Equation 2 ; that is: $\mathcal{E}_{G}=$ $\left\{(i, j) \in \mathcal{V} \times \mathcal{V}: a_{k i j} \neq 0\right.$, for at least one $\left.k \in\{1, \ldots, p\}\right\}$.

The contemporaneous network is defined as an undirected network $\mathcal{N}_{C}=\left(\mathcal{V}, \mathcal{E}_{C}\right)$, where an edge between $i$ and $j$ denotes that $i$ is partially correlated to $j$; that is, $\mathcal{E}_{C}=\left\{(i, j) \in \mathcal{V} \times \mathcal{V}: \rho^{i j} \neq 0\right\}$.

An alternative way to represent the dependence structure of the system using a single graph consists in simultaneously summarizing the lead/lag and contemporaneous relations of the process. We do this by introducing a partial correlation measure based on the long-run covariance matrix. This is inspired by the HAC literature (Den Haan \& Levin, 1996) and is a natural extension of the standard partial correlation network to serially dependent data. The long-run covariance matrix of the process $\mathbf{y}_{t}$ is defined as

$$
\Sigma_{L}=\lim _{M \rightarrow \infty} \frac{1}{M} \operatorname{cov}\left(\sum_{t=1}^{M} \mathbf{y}_{t}, \sum_{t=1}^{M} \mathbf{y}_{t}\right) .
$$

Equivalently, the long-run covariance may also be defined in terms of the sum of all autocovariance functions of the process $\Sigma_{L}=\sum_{h=-\infty}^{+\infty} \operatorname{cov}\left(\mathbf{y}_{t}, \mathbf{y}_{t-h}\right)$, which shows how $\Sigma_{L}$ synthesizes the linear dependencies of $\mathbf{y}_{t}$ at every lead and lag. Note that since the VAR is assumed to be stationary the sum above is well defined. The long-run covariance is therefore the spectral density matrix of $\mathbf{y}_{t}$ at zero frequency, which in the case of a VAR is given by

$$
\Sigma_{L}=\left(\mathbf{I}-\sum_{k=1}^{p} \mathbf{A}_{k}\right)^{-1} \mathbf{C}^{-1}\left(\mathbf{I}-\sum_{k=1}^{p} \mathbf{A}_{k}^{\prime}\right)^{-1} .
$$


We propose a network definition based on the partial correlations constructed on the basis of the long-run concentration matrix, which is defined as

$$
\mathbf{K}_{L}=\Sigma_{L}^{-1}=\left(\mathbf{I}-\sum_{k=1}^{p} \mathbf{A}_{k}^{\prime}\right) \mathbf{C}\left(\mathbf{I}-\sum_{k=1}^{p} \mathbf{A}_{k}\right) .
$$

This is also known as the zero-frequency partial spectral coherence (Dahlhaus, 2000; Davis et al., 2016). Note that $\mathbf{K}_{L}$ is factorized in a sandwich form determined by the term $\mathbf{I}-\sum_{k=1}^{p} \mathbf{A}_{k}$, which captures long-run dynamic relations of the system, and the term $\mathbf{C}$, which accounts for the contemporaneous dependence of the system innovations. We can then express long-run partial correlation coefficient for series $i$ and $j$ as a function of the entries $k_{L i j}$ of the long-run concentration matrix $\mathbf{K}_{L}$

$$
\rho_{L}^{i j}=-\frac{k_{L i j}}{\sqrt{k_{L i i} k_{L j j}}} .
$$

The long-run partial correlation network is then defined as an undirected network $\mathcal{N}_{L}=\left(\mathcal{V}, \mathcal{E}_{L}\right)$, where the set of edges $\mathcal{E}_{L}$ is defined as $\mathcal{E}_{L}=\left\{(i, j) \in \mathcal{V} \times \mathcal{V}: \rho_{L}^{i j} \neq 0\right\}$.

A number of comments on the model and network definitions we propose are in order. First, an important difference between the network modeling approach proposed here and other contributions in the literature is that we focus on representing the partial dependence structure of the panel. On the other hand, the contributions of Billio et al. (2012) and Diebold and Yllmaz (2014), among others, propose network definitions that measure the overall degree of dependence between series. The advantage of the approach proposed here is that it is robust to spurious correlation effects among the variables in the system. Moreover, the network definitions we propose can be seen as a natural extension for time series data of the popular partial correlation network models used in the statistics and graphical model literature.

Second, the network representations we propose are useful when the underlying dependence structure of the system is sparse. Sparsity assumptions (which are be spelled out precisely in what follows) are also key to carrying out inference in large-dimensional systems. It is important to acknowledge, however, that a number of contributions have criticized these types of assumptions on the grounds that they may not be appropriate for applications in economics and finance. Giannone, Lenza, and Primicieri (2018) analyze different economic datasets and conclude that, in general, sparsity is not a feature of the data and should be assumed only if there is enough a priori evidence in favor of predictive models with a small number of regressors.

Third, and related to the previous point, in this work we consider network analysis as a complement of factor analysis for the purpose of empirical applications. Indeed, an important case in which the assumption of sparsity is violated, is when the components of the panel are a function of a set of common factors (Bai, 2003; Forni, Hallin, Lippi, \& Reichlin, 2000; Stock \& Watson, 2002). In particular, it is straightforward to see that global common factors induce a fully interconnected network structure among the variables in the panel. ${ }^{1}$ Note also that the dependencies we are interested in, being conditional, cannot in general be captured by adding a small number of additional sectoral factors, as proposed for example by Foerster, Sarte, and Watson (2011). The presence of common factors in panels of volatilities, similar to those analyzed in this paper, is documented for example in Barigozzi, Brownlees, Gallo, and Veredas (2014) and Barigozzi and Hallin (2016). On the other hand, Barigozzi and Hallin (2017) show evidence of weak cross-sectional dependence once common factors are removed. As a consequence of these observations, we suggest that the influence of global and sectoral factors ought to be filtered out before carrying out network analysis. Specifically, our analysis in Section 4 shows that, even after controlling for global and sectoral factors in financial markets, network effects, in the form of conditional dependencies, still have an important predictive role.

Lastly, we point out that an alternative approach for the estimation of the long-run partial correlation network consists in estimating the long-run concentration matrix and then applying LASSO-type regularization (under the assumption of sparsity of $\mathbf{K}_{L}$ ). The advantage of estimating $\mathbf{K}_{L}$ directly is that one may rely on nonparametric long-run covariance estimators, which are consistent for a large class of data-generating processes. In practice, however, when it is known that two series have nonzero long-run (partial) correlation it is natural to ask whether this dependence arises because of lagged or contemporaneous effects (or both). To this extent a VAR is a natural modeling approach that allows us to disentangle these different channels.

\footnotetext{
${ }^{1}$ Consider an $n$-dimensional panel of time series $y_{i t}$ generated by a one-factor model $y_{i t}=\beta_{i} f_{t}+\epsilon_{i t}$, where $f_{t}$ and $\epsilon_{i t}$ are independent normals with zero mean and unit variance and $\epsilon_{i t}$ and $\epsilon_{j t}$ are independent for each $i \neq j$. Then the concentration matrix of the system is $\mathbf{K}=\mathbf{I}-\frac{1}{1+\beta^{\prime} \beta} \boldsymbol{\beta} \boldsymbol{\beta}^{\prime}$, where $\mathbf{I}$ is the identity matrix of size $n \times n$ and $\beta$ is an $n \times 1$ vector of factor loadings $\beta_{i}$. If the vector of factor loading does not contain zero entries then $\mathbf{K}$ is not sparse.
} 


\section{2 | Estimation}

We are interested in detecting and estimating the nonzero entries of the autoregressive matrices $\mathbf{A}_{k}$ and the concentration matrix C. A simple estimation approach for the sparse VAR would consist of using LASSO regression to estimate the autoregressive matrices $\mathbf{A}_{k}$ (as, for example, in Kock \& Callot, 2015), and then using a LASSO procedure on the residuals to estimate the concentration matrix $\mathbf{C}$ (as, for example, in Friedman et al., 2008; Peng et al., 2009). The analysis of properties of the second step estimator is, however, challenging. Moreover, the rate of convergence of the estimator of the concentration matrix $\mathbf{C}$ would depend on the rate of convergence of the estimator of the autoregressive matrices $\mathbf{A}_{k} .{ }^{2}$ In this work we propose an estimation approach that avoids these hurdles by estimating both sets of parameters jointly.

For ease of notation, we re-parametrize the VAR as a function of: (i) the coefficients $\alpha_{i j k}$ contained in an $n^{2} p$-dimensional vector $\boldsymbol{\alpha}$ which correspond to the autoregressive coefficients $a_{k i j}$ in Equation 1; (ii) the partial correlations $\rho^{i j}$ contained in an $n(n-1) / 2$-dimensional vector $\rho$ and defined in Equation 3; and (iii) the coefficients $c_{i i}$ contained in an $n$-dimensional vector $\mathbf{c}$ which correspond to the diagonal of the concentration matrix $\mathbf{C}$. Then, in scalar notation the parameters of our model are given by the VAR equations

$$
y_{i t}=\sum_{k=1}^{p} \sum_{j=1}^{n} \alpha_{i j k} y_{j t-k}+\epsilon_{i t}, \quad i=1, \ldots, n,
$$

and the contemporaneous equations (see Peng et al., 2009)

$$
\epsilon_{i t}=\sum_{\substack{h=1 \\ h \neq i}}^{n} \rho^{i h} \sqrt{\frac{c_{h h}}{c_{i i}}} \epsilon_{h t}+u_{i t}, \quad i=1, \ldots, n,
$$

where $u_{i t}$ is an error term uncorrelated with $\epsilon_{h t}$ for $i \neq h$.

In this section we define a novel LASSO-based estimator for the parameters of Equations 5 and 6 . We call the estimation algorithm nets (network estimator for time series) and we describe it in detail in the next section. The main feature of the proposed procedure is that it estimates the autoregressive parameters, $\boldsymbol{\alpha}$, and partial correlations, $\rho$, simultaneously, conditional on a pilot estimator of $\mathbf{c}$.

Consider the following regression representation of $y_{i t}$ as a function of the lags of all series as well as the contemporaneous realizations of all other series in the panel, that is:

$$
y_{i t}=\sum_{k=1}^{p} \sum_{j=1}^{n} \beta_{i j k} y_{j t-k}+\sum_{\substack{h=1 \\ h \neq i}}^{n} \gamma_{i h} y_{h t}+e_{i t},
$$

where $e_{i t}$ is an error term. It is straightforward to see that (see Lemma A1 in the Supporting Information Appendix) the $\beta_{i j k}$ and $\gamma_{i h}$ coefficients can be expressed as a function of the $\alpha_{i j k}, \rho^{i h}$, and $c_{i i}$ parameters. In particular, Equation 7 can be rewritten as

$$
y_{i t}=\sum_{k=1}^{p} \sum_{j=1}^{n} \underbrace{\left(\alpha_{i j k}-\sum_{\substack{l=1 \\ l \neq i}}^{n} \rho^{i l} \sqrt{\frac{c_{l l}}{c_{i i}}} \alpha_{l j k}\right)}_{\beta_{i j k}} y_{j t-k}+\sum_{\substack{h=1 \\ h \neq i}}^{\rho^{i h} \sqrt{\frac{c_{h h}}{c_{i i}}}} \underbrace{\underbrace{}_{i h}}_{\gamma_{h t}}+u_{i t} .
$$

Note that this result also shows that the errors $e_{i t}$ and $u_{i t}$ are the same. We denote by $\theta$ the vector of parameters of interest $\left(\boldsymbol{\alpha}^{\prime}, \boldsymbol{\rho}^{\prime}\right)^{\prime}$ of dimension $m=n^{2} p+n(n-1) / 2$. The regression representation in Equation 8 suggests associating the following quadratic loss function with the problem of determining $\theta$, conditional on $\mathbf{c}$ :

$$
\ell\left(\boldsymbol{\theta} ; \mathbf{y}_{t}, \mathbf{c}\right)=\sum_{i=1}^{n}\left(y_{i t}-\sum_{k=1}^{p} \sum_{j=1}^{n}\left(\alpha_{i j k}-\sum_{\substack{l=1 \\ l \neq i}}^{n} \rho^{i l} \sqrt{\frac{c_{l l}}{c_{i i}}} \alpha_{l j k}\right) y_{j t-k}-\sum_{\substack{h=1 \\ h \neq i}}^{n} \rho^{i h} \sqrt{\frac{c_{h h}}{c_{i i}}} y_{h t}\right)^{2} .
$$

\footnotetext{
${ }^{2}$ This is shown in a previous working paper version of this manuscript.
} 
If a sample of $T$ observations of the $\mathbf{y}_{t}$ process is available for $t=1, \ldots, T$, then we propose to estimate the model parameters using a LASSO-type estimator:

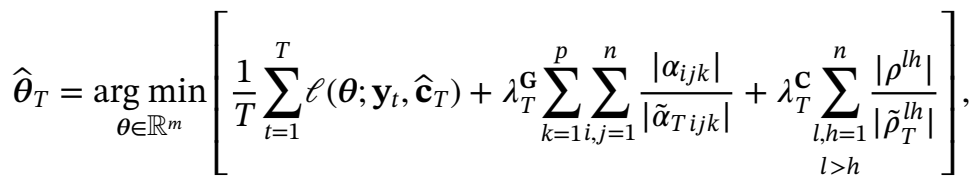

where $\lambda_{T}^{\mathbf{G}}>0$ and $\lambda_{T}^{\mathbf{C}}>0$ are the LASSO tuning parameters and $\widetilde{\boldsymbol{\alpha}}_{T}, \widetilde{\boldsymbol{\rho}}_{T}$ and $\widehat{\mathbf{c}}_{T}$ are pre-estimators of the $\boldsymbol{\alpha}, \boldsymbol{\rho}$, and $\mathbf{c}$ coefficients, respectively. Owing to the presence of autocorrelation in and across the components of $\mathbf{y}_{t}$, the regressors in Equation 8 are likely to be dependent; therefore we adopt here an adaptive LASSO penalty as originally proposed by Zou (2006) and then studied in a high-dimensional setting by Huang, Ma, and Zhang (2008) for independent data and by Kock (2016) for dependent data. If the sample size is sufficiently large, a natural pre-estimator of $\alpha$ is the least squares estimator of the VAR autoregressive matrices, while the pre-estimator of $\rho$ is the partial correlation estimator obtained from the sample covariance of the VAR residuals. Otherwise, if the sample size is not sufficiently large, a pre-estimator of $\boldsymbol{\alpha}$ could be obtained by estimating the autoregressive matrices via LASSO or ridge regression, while the pre-estimator of $\rho$ could be obtained from a shrinkage estimator of the residual covariance (Ledoit \& Wolf, 2004). A discussion of the implications of these choices for our theory is in Section 2.4. Last, a possible choice for the pre-estimator of $\mathbf{c}$ is the reciprocal of the variance of each series (Peng et al., 2009).

\subsection{The nets algorithm}

In this section we introduce the nets algorithm to solve the optimization problem of Equation 10. Note that the loss function in Equation 9 is not the standard quadratic loss function of a linear regression model and the standard LASSO algorithms cannot be applied. However, it is still possible to design a coordinate descent algorithm that can be used to minimize the objective function of Equation 10. The procedure we propose is a generalization of the space algorithm proposed by Peng et al. (2009) for the estimation of partial correlation networks, and it is a variation of the shooting algorithm by $\mathrm{Fu}(1998)$ typically used for LASSO optimization.

Additional notation is required to describe the algorithm. We begin by introducing the matrix representation of the model of Equation 8 obtained by stacking the time series in the panel. Let $\mathcal{Y}$ denote a $n T \times 1$ vector defined as $\left(y_{11}, \ldots, y_{1 T}, \ldots, y_{i 1}, \ldots, y_{i T}, \ldots, y_{n 1}, \ldots, y_{n T}\right)^{\prime} ;$ let $\boldsymbol{X}_{G}=\left(\boldsymbol{x}_{G 111}, \ldots, \boldsymbol{x}_{G i j k}, \ldots, \boldsymbol{x}_{G n n p}\right)$ be a $n T \times n^{2} p$ matrix with $(i, j, k)$ th column defined as

$$
\boldsymbol{x}_{G i j k}=(0, \ldots, 0, \underbrace{y_{j,-k}, \ldots, y_{j t-k}, \ldots, y_{j T-k}}_{i \text { th block }}, 0, \ldots, 0)^{\prime},
$$

and let $\mathcal{X}_{C}=\left(\boldsymbol{x}_{C 21}, \boldsymbol{x}_{C 31}, \boldsymbol{x}_{C 32}, \ldots, \boldsymbol{x}_{C i j}, \ldots, \boldsymbol{x}_{C n(n-1)}\right)$ be a $n T \times n(n-1) / 2$ matrix with $(i, j)$ th column defined as

$$
\boldsymbol{x}_{C i j}=(0, \ldots, 0, \underbrace{\sqrt{\frac{c_{j j}}{c_{i i}}}\left(y_{j 1}, \ldots, y_{j t}, \ldots, y_{j T}\right)}_{i \text {-th block }} 0, \ldots, \underbrace{\sqrt{\frac{c_{i i}}{c_{j j}}}\left(y_{i 1}, \ldots, y_{i t}, \ldots, y_{i T}\right)}_{j \text {-th block }} 0, \ldots, 0)^{\prime} .
$$

Then, it is straightforward to check that Model 8 can be represented as

$$
\mathcal{Y}=\mathcal{X}_{G} \beta(\alpha, \rho)+\mathcal{X}_{C} \rho+\boldsymbol{V}
$$

where $\boldsymbol{V}$ is a $n T \times 1$ vector of residuals and $\beta(\cdot, \cdot)$ denotes the function which maps the $\boldsymbol{\alpha}$ and $\rho$ parameter vectors onto the $\boldsymbol{\beta}$ parameter vector, whose components are given in Equation 7. Note that the parameter $\boldsymbol{\beta}$ and the matrix $\mathcal{X}_{C}$ depend implicitly on the parameter $\mathbf{c}$ and that in the estimation we set this to the pre-estimator $\widehat{\mathbf{c}}_{T}$. The dependence on $\mathbf{c}$ is suppressed in the notation for simplicity. In what follows it is convenient to introduce shorthand notation for the stacked vectors. Let $\boldsymbol{v}$ be an $n T \times 1$ stacked vector, then we use $v_{[i t]}$ to refer to the the element of the $i$ th block of $\boldsymbol{v}$.

The nets algorithm is an iterative coordinate descent procedure for the minimization of the objective function of Equation 10. Each iteration $s$ of the algorithm updates one component of the parameter vector $\boldsymbol{\theta}=\left(\boldsymbol{\alpha}^{\prime}, \boldsymbol{\rho}^{\prime}\right)^{\prime}$. The $\boldsymbol{\alpha}$ and $\boldsymbol{\rho}$ parameter estimates at iteration $s$ are denoted by $\hat{\boldsymbol{\alpha}}^{(s)}$ and $\hat{\boldsymbol{\rho}}^{(s)}$ respectively. We define the residual estimate at iteration $s$ as

$$
\widehat{\boldsymbol{v}}^{(s)}=\mathcal{Y}-\mathcal{X}_{G} \boldsymbol{\beta}\left(\hat{\boldsymbol{\alpha}}^{(s)}, \hat{\boldsymbol{\rho}}^{(s)}\right)-\mathcal{X}_{C} \hat{\boldsymbol{\rho}}^{(s)} .
$$

The algorithm iterates until convergence, which is checked at the end of each full cycle of updates of $\theta$. To describe the algorithm, it is useful to use two auxiliary $n T \times 1$ stacked vectors $\ddot{\boldsymbol{x}}$ and $\ddot{\boldsymbol{y}}$. The $\ddot{\boldsymbol{x}}$ vector denotes the regressors corresponding 
to the current coefficient being updated, while the $\ddot{\boldsymbol{y}}$ vector is the partial residual of the model with respect to all parameters besides the coefficient being currently updated (either $\alpha_{i j k}$ or $\rho^{i j}$ ). The $\alpha_{i j k}$ coefficient is updated as

$$
\hat{\alpha}_{i j k}^{(s)}=\operatorname{sign}\left(\ddot{\boldsymbol{y}}^{\prime} \ddot{\boldsymbol{x}}\right)\left(\left|\frac{\ddot{\boldsymbol{y}}^{\prime} \ddot{\boldsymbol{x}}}{\ddot{\boldsymbol{x}}^{\prime} \ddot{\boldsymbol{x}}}\right|-\frac{\lambda_{T}^{\mathbf{G}}}{\tilde{\alpha}_{i j k}} \frac{1}{\ddot{\boldsymbol{x}}^{\prime} \ddot{\boldsymbol{x}}}\right)_{+},
$$

where $\ddot{\boldsymbol{x}}$ and $\ddot{\boldsymbol{y}}$ are defined as

$$
\begin{aligned}
& \ddot{x}_{[l t]}= \begin{cases}y_{l t-k}, & \text { if } l=i \\
-\hat{\rho}^{i l(s-1)} \sqrt{\frac{\tilde{c}_{l l}}{\tilde{c}_{i i}}} y_{j t-k} & \text { otherwise }\end{cases} \\
& \ddot{y}_{[t t]}=\mathcal{V}_{[l t]}^{(s-1)}+\widehat{\alpha}_{i j k}^{(s-1)} \ddot{x}_{[l t]},
\end{aligned}
$$

for each $l=1, \ldots, n$ and $t=1, \ldots, T$. The $\rho^{i j}$ coefficient is updated as

$$
\hat{\rho}^{i j(s)}=\operatorname{sign}\left(\ddot{\boldsymbol{y}}^{\prime} \ddot{\boldsymbol{x}}\right)\left(\left|\frac{\ddot{\boldsymbol{y}}^{\prime} \ddot{\boldsymbol{x}}}{\ddot{\boldsymbol{x}}^{\prime} \ddot{\boldsymbol{x}}}\right|-\frac{\lambda_{T}^{\mathrm{c}}}{\tilde{\rho}^{i j}} \frac{1}{\ddot{\boldsymbol{x}}^{\prime} \ddot{\boldsymbol{x}}}\right)_{+},
$$

where $\ddot{\boldsymbol{x}}$ and $\ddot{\boldsymbol{y}}$ are defined as

$$
\begin{aligned}
& \ddot{x}_{[t]]}=\sqrt{\frac{\tilde{c}_{h h}}{\tilde{c}_{l l}}}\left(y_{h t}-\sum_{j=1}^{n} \sum_{k=1}^{p} \alpha_{h j k}^{(s-1)} y_{j t-k}\right) \\
& \ddot{y}_{[t]]}=\mathcal{V}_{[l t]}^{(s-1)}+\hat{\rho}^{i j(s-1) \ddot{x}_{[l t]},}
\end{aligned}
$$

for $(l, h)$ equal $(i, j)$ or $(j, i)$ and $t=1, \ldots, T$, and otherwise $\ddot{y}_{[l t]}$ and $\ddot{x}_{[t]}$ are set to zero. It is important to stress that the parameter vector $\theta$ contains $n^{2} p+\frac{n(n-1)}{2}$ elements, whose optimization would require large amounts of memory to be stored when the panel is large. On the other hand, the coordinate-wise minimization algorithm is appealing in this context in that it has modest storage requirements and can be applied in large-dimensional applications.

As far as the estimation of $\mathbf{c}$ is concerned, we follow the two-step iterative procedure proposed in Peng et al. (2009): (i) given an estimate of $\mathbf{c}$, we estimate the $\boldsymbol{\theta}$ parameter using nets; (ii) given an estimate $\mathbf{c}$ and an estimate of $\boldsymbol{\theta}$ we update the estimate of $\mathbf{c}$. Note that $c_{i i}$ is the reciprocal of the residual variance of Equation 8. These two steps are then iterated until convergence, which typically kicks in within very few iterations.

\section{4 | Theory}

We establish the estimation and selection consistency of our LASSO estimator. In this section we denote by $\boldsymbol{\theta}_{0}=\left(\boldsymbol{\alpha}_{0}^{\prime}, \boldsymbol{\rho}_{0}^{\prime}\right)^{\prime}$ and $\mathbf{c}_{0}$ the true value of the parameters, while their generic values are denoted by $\boldsymbol{\theta}=\left(\boldsymbol{\alpha}^{\prime}, \boldsymbol{\rho}^{\prime}\right)^{\prime}$ and $\mathbf{c}$. The estimator defined in Equation 10 can be equivalently formulated as

$$
\widehat{\boldsymbol{\theta}}_{T}=\underset{\boldsymbol{\theta} \in \mathbb{R}^{m}}{\arg \min } \mathcal{L}_{T}\left(\boldsymbol{\theta}, \widehat{\mathbf{c}}_{T}\right),
$$

where

$$
\mathcal{L}_{T}\left(\boldsymbol{\theta}, \widehat{\mathbf{c}}_{T}\right)=\left[\frac{1}{T} \sum_{t=1}^{T} \ell\left(\boldsymbol{\theta} ; \mathbf{y}_{t}, \widehat{\mathbf{c}}_{T}\right)+\lambda_{T} \sum_{i=1}^{m} w_{i}\left|\theta_{i}\right|\right],
$$

where $\lambda_{T}$ is the LASSO tuning parameter and $w_{i}$ are the adaptive LASSO weights. The specification of the weight is $w_{i}=C_{\bullet} /\left|\tilde{\theta}_{T i}\right|$, where $\tilde{\theta}_{T i}$ denotes the pre-estimator of the $\theta_{i}$ coefficient and $C_{\bullet}$. denotes a positive constant that is equal to $C_{\alpha}$ for the $\alpha$ coefficients and $C_{\rho}$ for the $\rho$ coefficients. Put differently, in the theoretical analysis of the estimator we assume that $\lambda_{T}^{\mathbf{G}}=\lambda_{T} C_{\alpha}$ and $\lambda_{T}^{\mathbf{C}}=\lambda_{T} C_{\rho}$, so that $\lambda_{T}^{\mathbf{G}} / \lambda_{T}^{\mathbf{C}}=O(1)$. Thus $\lambda_{T}$ controls the overall degree of shrinkage of the parameters of the model. We make the following assumptions.

Assumption 1. The $n$-dimensional random vector process $\mathbf{y}_{t}$ follows a $\operatorname{VAR}(p) \mathbf{y}_{t}=\sum_{k=1}^{p} \mathbf{A}_{0 k} \mathbf{y}_{t-k}+\boldsymbol{\epsilon}_{t}$, where $\boldsymbol{\epsilon}_{t} \sim$ i.i.d. $\left(\mathbf{0}, \mathbf{C}_{0}^{-1}\right)$. Moreover:

(a) $\operatorname{det}\left(\mathbf{I}-\sum_{k=1}^{p} \mathbf{A}_{0 k} z^{k}\right) \neq 0$ for any $|z| \leq 1$;

(b) there exists a constant $c>0$ such that $\mathrm{E}\left[\left|\epsilon_{i t}\right|^{k}\right] \leq k ! c^{k-2} \mathrm{E}\left[\epsilon_{i t}^{2}\right]<\infty$, for any $i=1, \ldots, n, t=1, \ldots, T$, $k=3,4, \ldots$;

(c) there exist couples of constants $\underline{M}_{0}, \bar{M}_{0}$ such that $0<\underline{M}_{0} \leq \mu_{\min }(\Sigma(\omega)) \leq \mu_{\max }(\Sigma(\omega)) \leq \bar{M}_{0}<\infty$, where $\Sigma(\omega)$ is the spectral density matrix of $\mathbf{y}_{t}$, defined for $\omega \in[-\pi, \pi]$, and $\underline{M}_{1}, \bar{M}_{1}$ such that $0<\underline{M}_{1} \leq \mu_{\min }\left(\mathbf{C}_{0}\right) \leq$ $\mu_{\max }\left(\mathbf{C}_{0}\right) \leq \bar{M}_{1}<\infty$. 
In part (a) we assume $\mathbf{y}_{t}$ to be generated by a stable VAR. In part (b) we assume a standard Cramér condition for the innovations. It is important to highlight that this implies the existence of all moments of the innovation distribution. This, in turn, implies that the innovation distribution is assumed to be light tailed, as in the case of Gaussian and sub-Gaussian distributions. It is then straightforward to see that, because of independence of the innovations, parts (a) and (b) together imply that $\mathbf{y}_{t}$ is a strong mixing process with moments also satisfying the Cramér condition (see, e.g., the comments in Fan $\&$ Yao, 2005, section 2.6). For such processes suitable Bernstein-type exponential inequalities for dependent processes apply (see, e.g., Bosq, 1996; Doukhan \& Neumann, 2007; Merlevède, Peligrad, \& Rio, 2011). Finally, in part (c) we assume positive definiteness of the spectral density matrix of $\mathbf{y}_{t}$ and of the precision matrix, $\mathbf{C}_{0}$, of the VAR innovations $\epsilon_{t}$. This guarantees that the population Granger and contemporaneous network are both well defined. Note that by assuming finite eigenvalues of spectral density we implicitly rule out the presence of a strong factor structure as the one assumed for example in Forni et al. (2000). However, this is not ruling out either cross-sectional or serial dependence, and weakly influential factors are still possible (Ando \& Bai, 2017; Onatski, 2012). That said, it is important to emphasize that when a high degree of collinearity is present in the data then LASSO estimation can be unstable (De Mol, Giannone, \& Reichlin, 2008). In these settings ridge estimation may be a better suited estimation strategy for large systems.

Additional notation has to be introduced before we can state the main result. The sets of nonzero parameters are denoted by $\mathcal{A}_{G}=\left\{(i, j, k): \alpha_{0 i j k} \neq 0\right\}, \mathcal{A}_{C}=\left\{(i, j): \rho_{0}^{i j} \neq 0\right\}$ and $\mathcal{A}=\mathcal{A}_{G} \cup \mathcal{A}_{C}$. The number of nonzero parameters in the model is $q_{T}=|\mathcal{A}|$. The set of zero parameters is then $\mathcal{A}^{c}$.

Assumption 2. Consider the pre-estimators $\widehat{\mathbf{c}}_{T}, \tilde{\boldsymbol{\theta}}_{T}=\left(\tilde{\boldsymbol{\alpha}}_{T}, \tilde{\boldsymbol{\rho}}_{T}\right)$. Then, for $T$ sufficiently large there exist constants $C_{1}, C_{2}, C_{3}>0$ such that, for any $\eta>0$, with probability at least $1-O\left(T^{-\eta}\right)$, we have (a) $\max _{1 \leq i \leq n}\left|\widehat{c}_{T i i}-c_{0 i i}\right| \leq$ $C_{1} \sqrt{\frac{\log T}{T}} ;$ (b) $\max _{i \in \mathcal{A}^{c}}\left|\tilde{\theta}_{T i}\right| \leq C_{2} \sqrt{\frac{\log T}{T}} ;$ (c) $\max _{i \in \mathcal{A}}\left|\tilde{\theta}_{T i}-\theta_{0 i}\right| \leq C_{3} \sqrt{q_{T} \frac{\log T}{T}}$.

Condition (a) is also required by Peng et al. (2009). Condition (b) is equivalent to the assumption made by Medeiros and Mendes (2016), while condition (c) is stronger (see also Huang et al., 2008). We discuss possible pre-estimators satisfying these conditions after stating our main result.

The following proposition establishes the estimation and selection consistency of our proposed estimator.

Proposition 1. (Selection consistency and oracle property). Suppose that, as $T \rightarrow \infty, q_{T}=o\left(\sqrt{\frac{T}{\log T}}\right), \lambda_{T} \sqrt{\frac{T}{\log T}} \rightarrow$ $\infty, \sqrt{q_{T}} \lambda_{T}=o(1), \sqrt{q_{T}} \sqrt{\frac{\log T}{T}}=o\left(\lambda_{T}\right), n=O\left(T^{\zeta}\right)$ for some $\zeta>0$. Let also $\left\{s_{T}\right\}$ be a positive signal sequence of real numbers such that for any $i \in \mathcal{A}$ we have $\left|\theta_{0 i}\right| \geq s_{T}$ and such that $\frac{s_{T}}{\sqrt{q_{T}} \lambda_{T}} \rightarrow \infty$. Then, under Assumptions 1 and 2, for any $\eta>0, \widehat{\boldsymbol{\theta}}_{T}$ exists with probability at least $1-O\left(T^{-\eta}\right)$. Moreover: $(a) \operatorname{Pr}\left(\hat{\theta}_{T i}=0\right) \geq 1-O\left(T^{-\eta}\right)$, for any $i \in \mathcal{A}^{c} ;(b)$ there exists a constant $\kappa>0$ such that

$$
\operatorname{Pr}\left(\left\|\widehat{\boldsymbol{\theta}}_{T}-\boldsymbol{\theta}_{0}\right\| \leq \kappa \sqrt{q_{T}} \lambda_{T}\right) \geq 1-O\left(T^{-\eta}\right),
$$

and $\operatorname{Pr}\left(\operatorname{sign}\left(\hat{\theta}_{T i}\right)=\operatorname{sign}\left(\theta_{0 i}\right)\right) \geq 1-O\left(T^{-\eta}\right)$, for any $i \in \mathcal{A}$.

Note that, since we assume $q_{T}=o\left(\sqrt{\frac{T}{\log T}}\right)$, then condition (c) in Assumption 2 requires a vanishing bias for the pre-estimator. When the number of parameters to be estimated is small relative to $T$, then the least squares estimator of $\mathbf{A}_{0 k}$ and the sample covariance estimator of $\mathbf{C}_{0}$ can be used to build consistent pre-estimators of $\boldsymbol{\theta}_{0}$, and Assumption 2 is satisfied. When the number of parameters is large relative to $T$, LASSO and ridge are natural pre-estimators of $\mathbf{A}_{0 k}$. These do not automatically satisfy condition (c), as they may have a nonvanishing bias. In this case, additional conditions on the degree of penalization of the pre-estimators and on $\lambda_{T}$ and $q_{T}$ are needed. For LASSO those conditions would be similar to those in the work by Medeiros and Mendes (2016), to which we refer for details. For ridge, if we denote by $\lambda_{T}^{\text {ridge }}$ the parameter controlling the degree of penalization, it can be seen that the bias is $O\left(\sqrt{q_{T}} \lambda_{T}^{\text {ridge }}\right.$ ) (Bühlmann, 2013; Shao \& Deng, 2012) and. in this case, inspection of our proofs shows that, as long as $q_{T} \lambda_{T}^{\text {ridge }} \lambda_{T}=o(1)$, consistent estimators can still be obtained. Similar arguments apply for regularized pre-estimators of $\mathbf{C}_{0}$.

Proposition 1 implies the consistency of the networks estimators.

Corollary 1. Define as $\widehat{\alpha}_{T i j k}$ the generic entry of $\widehat{\boldsymbol{\alpha}}_{T}$ and as $\hat{\rho}_{T}^{i j}$ the generic entry of $\hat{\boldsymbol{\rho}}_{T}$, and define the estimated edges' sets of the Granger and contemporaneous networks as $\hat{\mathcal{E}}_{G T}=\left\{(i, j) \in \mathcal{V} \times \mathcal{V}: \widehat{\alpha}_{T i j k} \neq 0\right.$, for at least one $\left.k \in\{1, \ldots, p\}\right\}$, and $\hat{\mathcal{E}}_{C T}=\left\{(i, j) \in \mathcal{V} \times \mathcal{V}: \hat{\rho}_{T}^{i j} \neq 0\right\}$. Then, under the same Assumptions of Proposition 1, for T sufficiently large and any $\eta>0$, we have $\operatorname{Pr}\left(\hat{\mathcal{E}}_{G T}=\mathcal{E}_{G}\right) \geq 1-O\left(T^{-\eta}\right)$, and $\operatorname{Pr}\left(\hat{\mathcal{E}}_{C T}=\mathcal{E}_{C}\right) \geq 1-O\left(T^{-\eta}\right)$. 
Given the asymptotic conditions on the number of non-zero coefficients, $q_{T}$, the worst-case scenario is when it is almost in the order of $\sqrt{\frac{T}{\log T}}$. In that case $\lambda_{T}$ needs to be nearly in the order of $T^{-1 / 4}$ to achieve consistency. On the other hand, for the best-case scenario, that is when $q_{T}=O(1)$ (for example, when the dimension $n$ is fixed), then the order of $\lambda_{T}$ can be nearly as small as $T^{-1 / 2}$ (within a factor of $\log T$ ). Consequently, the $L_{2}$-norm distance of the estimator from the true parameter is in the order of $\sqrt{\frac{\log T}{T}}$, with probability at least $1-O\left(T^{-\eta}\right)$.

\section{3 | SIMULATION STUDY}

In this section we analyze the properties of our estimator using simulated data. The exercise consists in simulating a large sparse VAR process and then using the nets algorithm to estimate it.

We simulate an $n=100$-dimensional sparse VAR(1). Note that the total number of parameters in this system is 15,050 . The sparse autoregressive matrix $\mathbf{A}_{1}$ and concentration matrix $\mathbf{C}$ are obtained from an Erdös-Renyi random graph model. The Erdös-Renyi model is a graph $(\mathcal{V}, \mathcal{E})$ defined over a fixed set of vertices $\mathcal{V}$ and a random set of edges $\mathcal{E}$. The existence of an edge between vertices $i$ and $j$ is determined by a Bernoulli trial with probability $p$ that is independent of all other edges. The Erdös-Renyi random graph is said to be directed or undirected depending on whether the underlying edge set $\mathcal{E}$ is directed or not. In our simulation setting we begin by simulating a directed and an undirected Erdös-Renyi random graphs over a set of $n$ vertices $\mathcal{V}$ denoted, respectively, by $\mathcal{G}_{1}=\left(\mathcal{V}, \mathcal{E}_{1}\right)$ and $\mathcal{G}_{2}=\left(\mathcal{V}, \mathcal{E}_{2}\right)$. The probability of a link $p$ is set to $1 / n$ in both graphs. The $\mathbf{A}_{1}$ matrix is then constructed on the basis of a directed Erdös-Renyi model $\mathcal{G}_{1}$ as

$$
\boldsymbol{a}_{1 i j}= \begin{cases}0.275 & \text { if }(i, j) \in \mathcal{E}_{1} \\ 0 & \text { otherwise }\end{cases}
$$

where $\mathcal{E}_{1}$ is the set of (directed) edges of $\mathcal{G}_{1}$. The concentration matrix $\mathbf{C}$ is constructed on the basis of an undirected Erdös-Renyi model $\mathcal{G}_{2}$ :

$$
\boldsymbol{c}_{i j}= \begin{cases}-\frac{1}{\sqrt{d_{i} d_{j}}} & i \neq j \text { and }(i, j) \in \mathcal{E}_{2} \\ 1.5 & i=j \\ 0 & \text { otherwise, }\end{cases}
$$

where $\mathcal{E}_{2}$ is the set of (undirected) edges of $\mathcal{G}_{2}$ and $d_{i}$ denotes the degree of vertex $i$ in this graph. Note that the simulation is designed in a way such that the sparsity structure of the Granger and contemporaneous networks of the VAR coincide with that of the two random graphs $\mathcal{G}_{1}$ and $\mathcal{G}_{2}$. Also, the specification guarantees that the VAR is stable and that the concentration matrix is positive definite. We report in Figure 1 the plot of the Granger and contemporaneous networks associated with a randomly chosen realization of the model. Note that despite the networks being sparse (in the sense that the expected number of links is $O(n)$ ), they are almost fully interconnected. We simulate samples of different sizes from the sparse VAR(1) we just described $(T=250,500,750$ and 1000) and then use the net s algorithm to estimate the model. For simplicity, the tuning parameters $\lambda_{T}^{\mathbf{G}}$ and $\lambda_{T}^{\mathbf{C}}$ are set equal to a common shrinkage tuning parameter $\lambda_{T}$. Our LASSO estimator requires pre-estimators of the $\alpha$ and $\rho$ parameters to construct the LASSO penalty weights. The pre-estimator of $\boldsymbol{\alpha}$ is the least squares estimator of the VAR(1) autoregressive matrix, while the pre-estimator of $\rho$ is the partial correlation estimator obtained from the sample covariance of the VAR(1) residuals. Last, we initialize $\mathbf{c}$ using the reciprocal of the sample variances of each series. The model is then estimated over a range of values of the common shrinkage tuning parameter $\lambda_{T}$.

The simulation is replicated 1,000 times and the quality of the nets estimator is measured on the basis of the mean squared error (MSE) and the receiver operating characteristic (ROC) curve, which is the plot of the false discovery rate (FDR) of the estimator versus the true positive rate (TPR). We report in the left-hand panel of Figure 2 the MSE of the nets estimator as a function of the tuning parameter $\lambda_{T}$ for the sample size $T$ equal to 500,750 and 1,000. ${ }^{3}$ The picture displays the typical profile of shrinkage type estimators; that is, the MSE is a convex function of the tuning parameter, and as the sample size increases the MSE of the estimator decreases. The right-hand panel of Figure 2 reports the ROC curve associated with the nets estimator for the sample size $T$ equal to 250, 500, 750, and 1,000. Recall that the FDR is defined as the ratio of incorrectly detected nonzero parameters over the total number of zero parameters, while TPR is defined as the ratio of correctly detected nonzero parameters over the total number of nonzero parameters. Note that the

\footnotetext{
${ }^{3}$ We omit from the picture for $T=250$ because of scaling issues.
} 

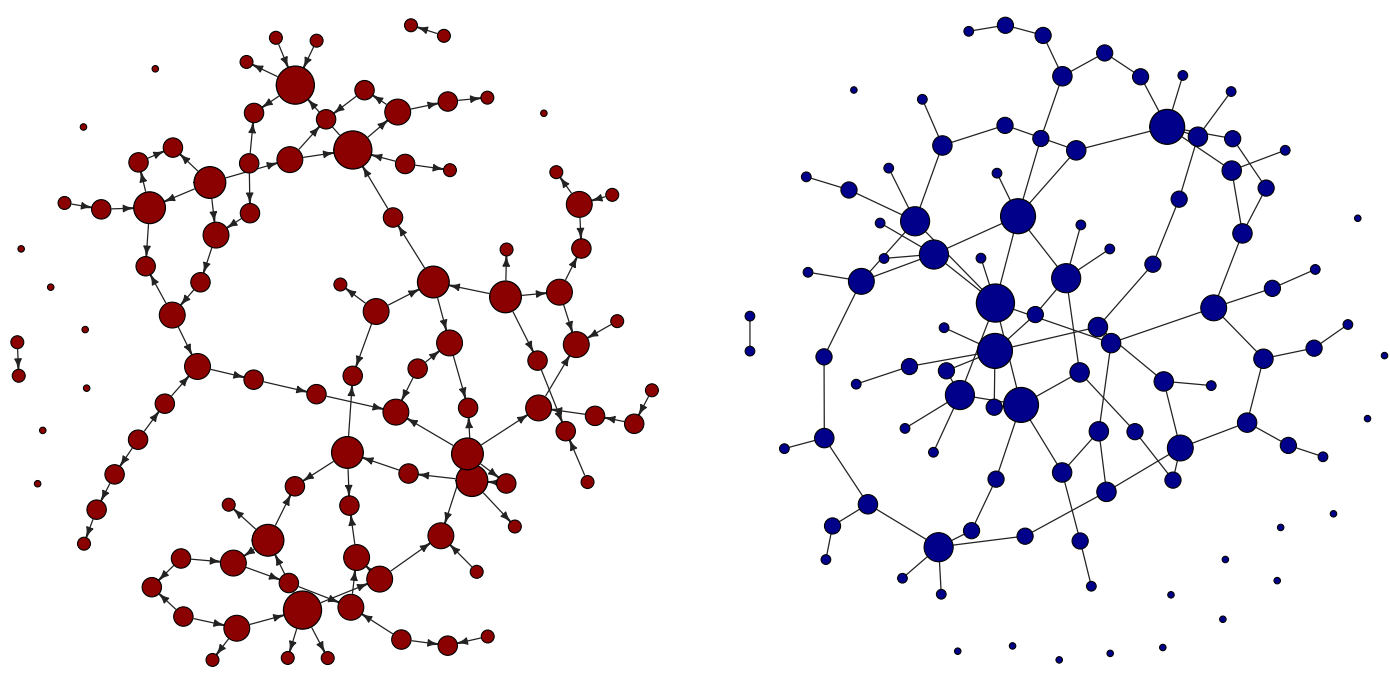

FIGURE 1 Simulated Granger and contemporaneous networks. The figure displays realizations of the Erdös-Renyi random graph models used in the simulation exercise. The left-hand picture displays a directed Erdös-Renyi graph used to generate the autoregressive matrix A; the right-hand picture displays an undirected Erdös-Renyi random graph used to generate the contemporaneous concentration matrix $\mathbf{C}$ [Colour figure can be viewed at wileyonlinelibrary.com]
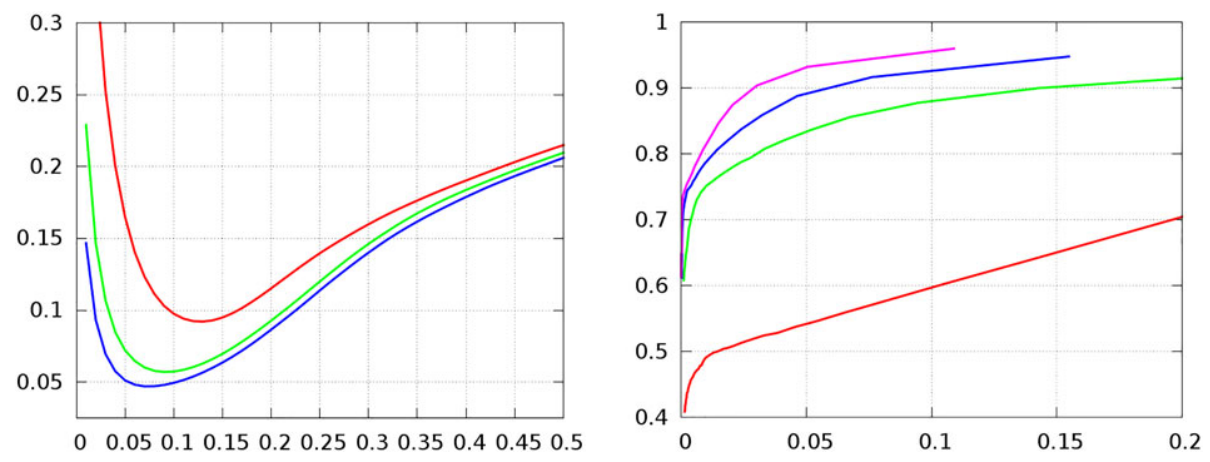

FIGURE 2 Simulation study. The left-hand picture displays the MSE (multiplied by 100) of the nets estimator as a function of the tuning parameter $\lambda_{T}$ for (from top to bottom) $T=500,750,1,000$. The right-hand picture displays the ROC curve of the net $\mathrm{s}$ estimator for (from bottom to top) $T=250,500,750,1,000$ [Colour figure can be viewed at wileyonlinelibrary.com]

TABLE 1 Simulation study

\begin{tabular}{|c|c|c|c|c|c|c|c|}
\hline \multirow[b]{2}{*}{$T$} & \multicolumn{2}{|c|}{ FDR $=1 \%$} & \multicolumn{2}{|c|}{$\mathrm{FDR}=\mathbf{5 \%}$} & \multicolumn{2}{|c|}{ FDR $=10 \%$} & \multirow{2}{*}{$\begin{array}{l}\text { MSE } \\
\text { pre-estimator }\end{array}$} \\
\hline & $\overline{\text { TPR }}$ & MSE & $\overline{\text { TPR }}$ & MSE & $\overline{\text { TPR }}$ & MSE & \\
\hline 250 & 0.49 & 0.32 & 0.55 & 0.31 & 0.60 & 0.34 & 5.33 \\
\hline 500 & 0.75 & 0.10 & 0.84 & 0.17 & 0.88 & 0.25 & 1.45 \\
\hline 750 & 0.79 & 0.06 & 0.89 & 0.11 & 0.92 & 0.15 & 0.81 \\
\hline 1,000 & 0.82 & 0.05 & 0.93 & 0.09 & 0.96 & 0.15 & 0.55 \\
\hline
\end{tabular}

Note. The table reports the results of the simulation exercise for different values of the sample size $T$. The table reports the true positive rate (TPR) and the MSE of the nets estimator when the false discovery rate (FDR) is controlled at the $1 \%, 5 \%$, and $10 \%$ levels. The last column of the table shows the MSE of the pre-estimator.

penalization coefficient determines the FDR and TPR properties of the estimator: when $\lambda_{T}$ is small (large), the proportion of type 1 errors is high (low), whereas the proportion of type 2 errors is low (high). The curves show that as the sample size $T$ increases the performance of the nets estimator, as measured by the area underneath the ROC curve, increases steadily. In Table 1 we report detailed results on the MSE and TPR of the nets estimator when the FDR is controlled at $1 \%, 5 \%$, and 10\%. For comparison purposes, the table also reports the MSE of the pre-estimator. The MSE of the nets 
estimator decreases steadily as the sample size get larger. When the sample size is 250 the efficiency gains with respect to the pre-estimator are substantial. As the sample size increases, the pre-estimator becomes progressively more efficient relative to the LASSO estimator; however, the efficiency gain of nets is still large. As far as the TPR is concerned, the table shows that when the TPR is controlled at $1 \%, 5 \%$ and $10 \%$ levels, the procedure has a fair amount of power even when the sample size $T$ is 250 , and that as the sample size increases power rises steadily. In particular, the power is roughly around $80 \%$ when the sample size is 750 and the FDR is controlled at the $1 \%$ level. Overall, the simulation results convey that the net s algorithm performs satisfactorily, and that the gains with respect to the traditional estimator can be large for sparse VAR systems.

\section{EMPIRICAL APPLICATION}

We use the methodology introduced in this work to study interconnectedness in a panel of volatility measures. The application is close in spirit to, among others, the research of Diebold and Yllmaz (2009, 2014, 2015) and Engle et al. (2012). We consider a panel of 90 US blue chips across different industry sectors. A list of company names and industry groups is given in Table C-1 in the Supporting Information Appendix. Our sample spans January 2, 2004, to December 31, 2015, which corresponds to 3,021 trading days. During this sample period most of the stocks in the list have been part of the S\&P 100 index. Following Diebold and Yllmaz (2015) we measure volatility using the high-low range (Parkinson, 1980):

$$
\tilde{\sigma}_{i t}^{2}=0.361\left(p_{i t}^{\text {high }}-p_{i t}^{\text {low }}\right)^{2}
$$

where $p_{i t}^{\text {high }}$ and $p_{i t}^{\text {low }}$ denote, respectively, the maximum and minimum log-price of stock $i$ on day $t^{4}$

We focus on analyzing volatility interconnectedness conditional on market-wide and sector-specific volatility factors. There is a large literature documenting evidence of a factor structure in volatility (see, among others, Barigozzi et al., 2014; Barigozzi \& Hallin, 2016). As previously pointed out, it is straightforward to check that when common factors are present the dependence structure of the data is not sparse. To this extent, we study the interconnectedness of the residuals of the regression:

$$
\log \tilde{\sigma}_{i t}^{2}=\beta_{0}+\beta_{1} \log \tilde{\sigma}_{m t}^{2}+\beta_{2} \log \tilde{\sigma}_{s t}^{2}+z_{i t},
$$

where $\tilde{\sigma}_{m t}^{2}$ and $\tilde{\sigma}_{s t}^{2}$ denote, respectively, market-wide and a sector-specific volatility factors. Thus with the notation used in the previous sections we have $z_{i t}=y_{i t}$. The market and sectoral volatilities are measured using the high-low range estimator applied to the S\&P 500 index and the SPDR sectoral indices of S\&P 500. ${ }^{5}$ The residuals are obtained after estimating the model by least squares. In what follows we refer to the volatility residual panel as the volatility panel for short.

Table 2 reports summary statistics on the variance, kurtosis, autocorrelation, average cross-correlation, and average cross-autocorrelation of order one for the volatility residuals. Moreover, in Figure 3 we show the heat maps of the sample autocorrelation matrix of order one and the sample correlation matrix. We note that after netting out the factors the volatility residuals still exhibit autocorrelation. It is important to emphasize that while the raw volatility measure exhibits long range dependence, the volatility residuals exhibit a considerably weaker autocorrelation structure. In a way, the volatility residuals can be thought of as the short-run idiosyncratic volatility component of a volatility component model (Barigozzi et al., 2014; Wang \& Ghysels, 2015). Inspection of the average correlations and the heat maps shows that contemporaneous and lagged cross-correlation is still present in the volatility residuals. Interestingly, tickers in the same industry still exhibit a moderate degree of correlation even after conditioning on the sectoral factors.

\section{1 | In-sample estimation results}

We analyze the panel of volatility measures using the net s algorithm over the entire sample. The order of the VAR model $p$ is set to one. The pre-estimator of the $\boldsymbol{\alpha}$ parameters is the least squares estimator of the VAR(1) autoregressive matrix, while the pre-estimator of the $\rho$ parameters is the partial correlation estimator obtained from the sample covariance of the

${ }^{4}$ Several advanced estimators of volatility based on high-frequency data have been proposed over the last years (Andersen, Bollerslev, Diebold, \& Labys, 2003). However, a number of contributions have pointed out that simple estimators, like the high-low range, perform satisfactorily (see also Alizadeh, Brandt, \& Diebold, 2002; Brownlees \& Gallo, 2010).

${ }^{5}$ The SPDR sectoral indices of the S\&P 500 we use are Consumer Discretionary (XLY), Consumer Staples (XLP), Energy (XLE), Financials (XLF), Health Care (XLV), Industrials (XLI), Materials (XLB), Technology (XLK), and Utilities (XLU). 
TABLE 2 Descriptive stats

\begin{tabular}{lllllllllll} 
& Disc & Stap & Ener & Fin & Heal & Ind & Tech & Mat & Util & All \\
\hline Variance & 0.064 & 0.049 & 0.042 & 0.064 & 0.059 & 0.050 & 0.059 & 0.064 & 0.041 & 0.056 \\
Kurtosis & 4.401 & 5.434 & 4.981 & 4.929 & 4.993 & 4.676 & 4.467 & 4.413 & 5.740 & 4.807 \\
$\rho_{1}$ & 0.260 & 0.231 & 0.206 & 0.309 & 0.254 & 0.222 & 0.257 & 0.320 & 0.193 & 0.253 \\
$\rho_{5}$ & 0.170 & 0.137 & 0.144 & 0.241 & 0.156 & 0.133 & 0.163 & 0.237 & 0.120 & 0.168 \\
$\rho_{22}$ & 0.142 & 0.104 & 0.123 & 0.183 & 0.118 & 0.109 & 0.120 & 0.202 & 0.075 & 0.132 \\
$\rho_{0, \text { others }}$ & 0.091 & 0.089 & 0.063 & 0.077 & 0.087 & 0.098 & 0.080 & 0.073 & 0.069 & 0.083 \\
$\rho_{1, \text { others }}$ & 0.045 & 0.048 & 0.024 & 0.028 & 0.046 & 0.045 & 0.042 & 0.034 & 0.034 & 0.039 \\
\hline
\end{tabular}

Note. The table reports average descriptive statistics over the industry sectors and the entire panel. The set of descriptive statistics considered contains the sample variance, kurtosis, autocorrelation of order 1,5 , and 22 , the average contemporaneous correlation with all other tickers, and the average order 1 autocorrelation with all other tickers.
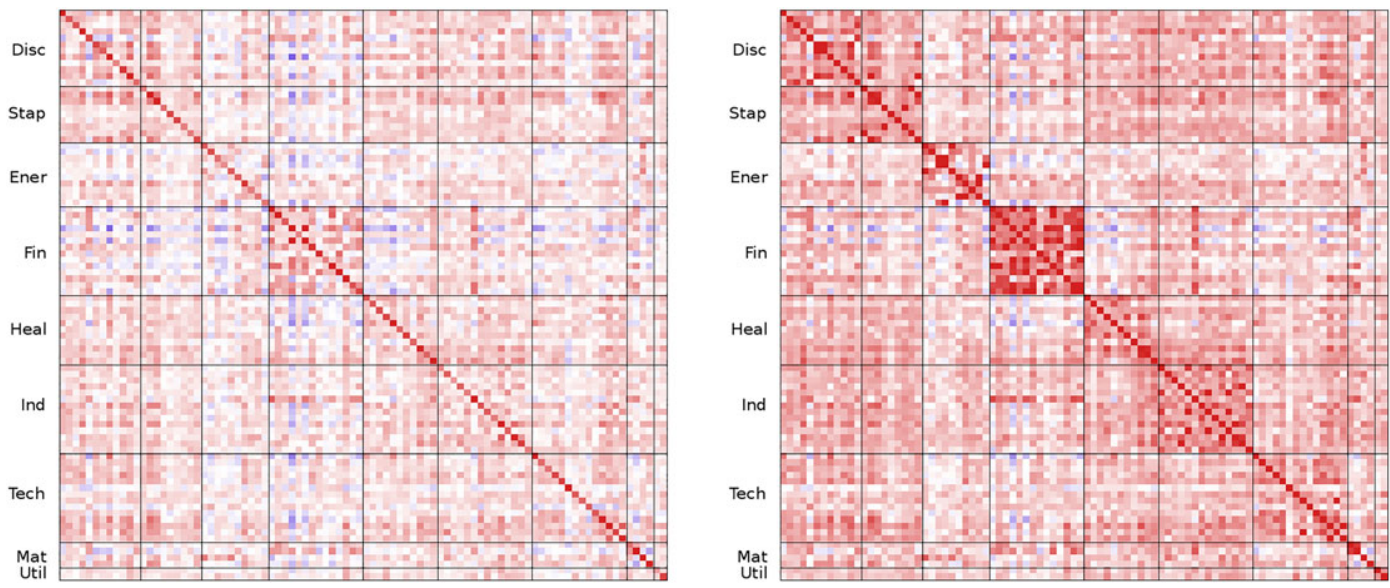

FIGURE 3 Autocorrelation and correlation heat maps. The figure displays the heat map of the sample autocorrelation (left) and sample correlation matrices (right) of the residuals of the regression in Equation 13 [Colour figure can be viewed at wileyonlinelibrary.com]

VAR(1) residuals. Lastly, we initialize $\mathbf{c}$ using the reciprocal of the sample variances of each series. The penalties $\lambda_{T}^{\mathbf{G}}$ and $\lambda_{T}^{\mathrm{C}}$ are determined by a cross-validation procedure. We split the entire sample into estimation and validation samples. The estimation sample corresponds to the first $75 \%$ of the entire sample and the validation sample to the last $25 \%$. For given values of the tuning parameters, we first estimate the model in the estimation sample and then compute the residual sum of squares (RSS) in the validation sample. We perform these steps over a grid of $\lambda_{T}^{\mathbf{G}}$ and $\lambda_{T}^{\mathbf{C}}$ values and then choose the optimal tuning parameters as those that minimize the validation RSS. We then estimate the model over the entire sample using the optimal value of the tuning parameters.

We report the estimated Granger and contemporaneous networks in Figure 4. In the Granger network plot the diameter of each vertex is proportional to the out-degree (the number of nonzero spillover effects toward others), whereas in the contemporaneous network the diameter is proportional to the degree. In both plots we use the vertex color to denote the different industry groups. We exclude from the graphs the vertices that do not have any connections, which is one ticker in the Granger network and seven tickers in the contemporaneous network.

Table 3 reports the sum of the degrees of the vertices in the Granger and contemporaneous networks over the entire panel and individual sectors. The estimated Granger volatility network has approximately $3 \%$ of the total edges, whereas the contemporaneous volatility network has approximately $4 \%$ of the total edges. The estimated networks share some common features. For instance, the number of industry linkages of the two networks are highly correlated and the financial sector is, in particular, the sector that accounts for most linkages.

We compute an in-sample $R^{2}$ type goodness-of-fit criterion for each series in the panel to summarize the amount of variability explained by the sparse VAR, which is defined as the proportion of variance explained by the regression equation (8). Table 3 reports the average of the $R^{2}$ index over the entire panel as well as the individual sectors. The index 

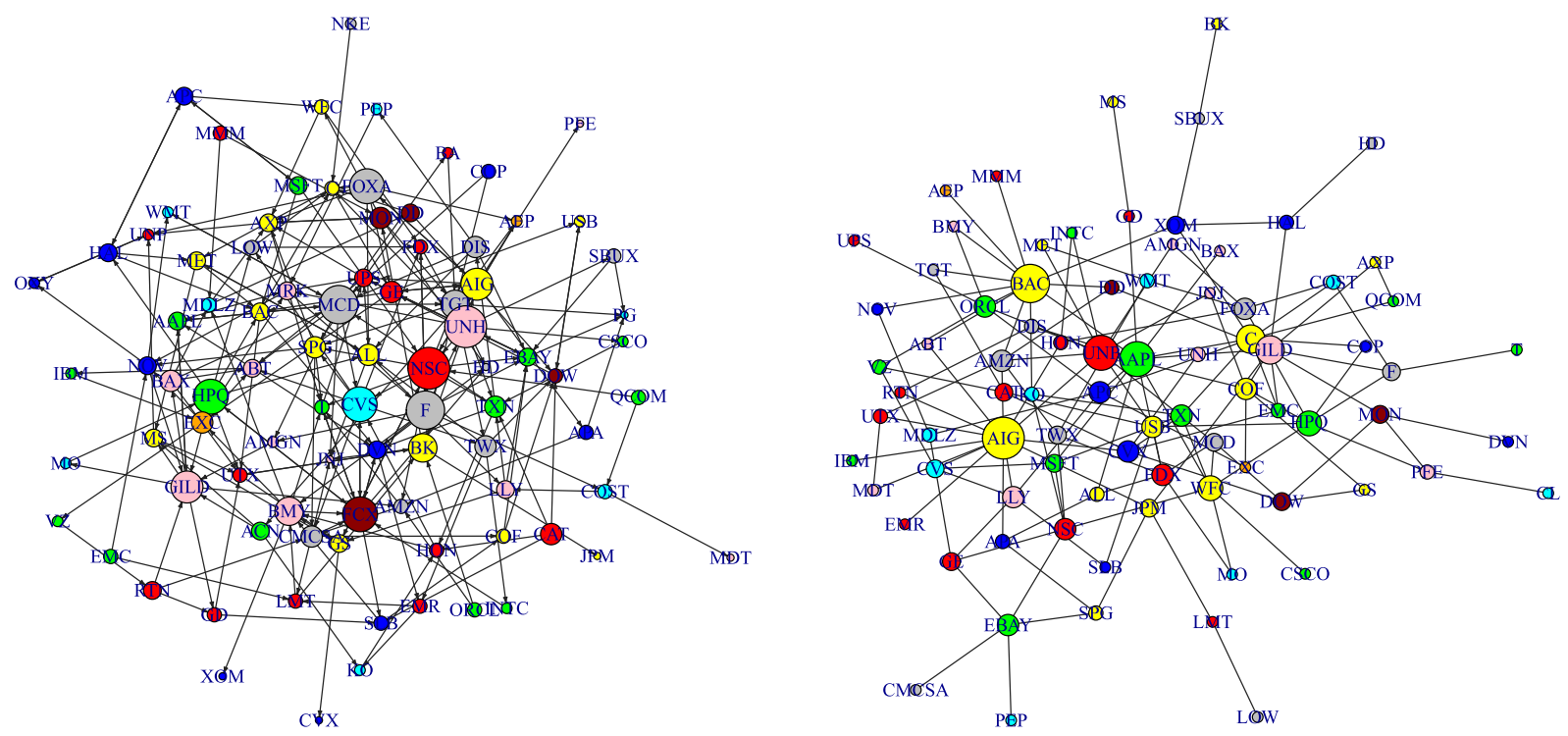

FIGURE 4 S\&P 100 volatility Granger and contemporaneous networks. The figure displays the estimated Granger and contemporaneous networks. The size of the vertices is proportional to their degree and the color of the vertices depends on their industry sector [Colour figure can be viewed at wileyonlinelibrary.com]

TABLE 3 Network estimation summary

\begin{tabular}{lllllllllll} 
& Disc & Stap & Ener & Fin & Heal & Ind & Tech & Mat & Util & All \\
Granger links & 47 & 15 & 19 & 41 & 34 & 39 & 35 & 16 & 5 & 251 \\
Contemporaneous links & 33 & 20 & 29 & 71 & 30 & 46 & 51 & 11 & 3 & 294 \\
nets $R_{\text {is }}^{2}$ & 22.5 & 18.6 & 18.9 & 32.2 & 18.9 & 24.4 & 20.0 & 19.5 & 11.0 & 22.3 \\
Factor $R_{\text {is }}^{2}$ & 45.3 & 37.2 & 42.7 & 56.6 & 33.6 & 51.3 & 39.1 & 45.9 & 36.8 & 44.4 \\
Sector $R_{\text {is }}^{2}$ & 7.3 & 9.6 & 25.3 & 16.1 & 9.0 & 5.8 & 8.4 & 10.6 & 25.8 & 11.6 \\
\hline
\end{tabular}

Note. The table reports summary estimation results over the industry sectors and the entire panel. The first row of the table reports the outer degree of the Granger network, the second row reports the degree of the contemporaneous network, and the third row reports the (in-sample) average $R_{\text {is }}^{2}$ of the nets regression. For comparison, the table reports in the fourth and fifth rows the average (in-sample) factor $R_{\text {is }}^{2}$ and (in-sample) sector $R_{\text {is }}^{2}$, respectively.

has a strong positive correlation with the number of linkages in each sector and is, on average, around $22 \%$. For comparison purposes, Table 3 also reports in-sample factor and sectoral $R^{2}$. The factor $R^{2}$ is defined as the $R^{2}$ obtained by regressing the volatility measure on the market factor and the sector $R^{2}$ is defined as the $R^{2}$ obtained by regressing the volatility measure on the market wide and sector factor minus the factor $R^{2}$. The market and sector factors account for most of the variability in the series, which is roughly 56\%. A back-of-the-envelope computation shows that the networks explain around an additional $11 \%$ of the overall variability, which roughly matches the amount of variability explained by sectoral factors.

In order to obtain better insights into the industry linkages in Table 4 we report the total number of links between industry groups. It is interesting to note that after conditioning on the sectoral factors there are still a moderate number of interconnections between firms within the same industry. The table also shows that firms in the financial sector in particular have a high degree of interconnectedness across industries. In Figure 5 we report the degree distribution of the estimated networks and the distribution of the nonzero $\alpha$ and $\rho$ coefficients. As far as the degree distribution is concerned, the number of connections has a high degree of heterogeneity in the cross-section. In particular, in the contemporaneous network the most interconnected tickers account for a large number of connections relative to the total. The histogram of the nonzero coefficients shows that the majority of the coefficients are positive and that positive coefficients are, on average, larger than the negative ones.

We rank the firms in the panel on the basis of their influence in the Granger and contemporaneous networks. We measure the influence of series $j$ in the Granger and contemporaneous networks using, respectively, the indices $\sum_{i \neq j}^{N}\left|\widehat{\alpha}_{i j 1}\right|$ and $\sum_{i \neq j}^{N}\left|\hat{\rho}^{i j}\right|$. We report the top 10 most influential tickers of the Granger and contemporaneous networks according to 
TABLE 4 Sectoral linkages

\begin{tabular}{|c|c|c|c|c|c|c|c|c|c|}
\hline & Disc & Stap & Ener & Fin & Heal & Ind & Tech & Mat & Util \\
\hline \multicolumn{10}{|c|}{ Granger component } \\
\hline Disc & 17.2 & 31.6 & 4.8 & 17.1 & 27.3 & 17.5 & 21.4 & 13.3 & 40.0 \\
\hline Stap & 13.8 & 0.0 & 0.0 & 2.4 & 9.1 & 5.0 & 3.6 & 6.7 & 0.0 \\
\hline Ener & 6.9 & 10.5 & 19.0 & 9.8 & 3.0 & 7.5 & 7.1 & 6.7 & 0.0 \\
\hline Fin & 17.2 & 0.0 & 19.0 & 14.6 & 9.1 & 25.0 & 14.3 & 40.0 & 40.0 \\
\hline Heal & 17.2 & 15.8 & 14.3 & 14.6 & 15.2 & 15.0 & 28.6 & 6.7 & 20.0 \\
\hline Ind & 6.9 & 21.1 & 9.5 & 19.5 & 9.1 & 12.5 & 14.3 & 6.7 & 0.0 \\
\hline Tech & 13.8 & 15.8 & 19.0 & 12.2 & 15.2 & 15.0 & 3.6 & 20.0 & 0.0 \\
\hline Mat & 6.9 & 5.3 & 9.5 & 9.8 & 3.0 & 2.5 & 7.1 & 0.0 & 0.0 \\
\hline Util & 0.0 & 0.0 & 4.8 & م & 01 & م & 0 & 0 & 00 \\
\hline \multicolumn{10}{|c|}{ Contemporaneous component } \\
\hline Disc & 5.3 & 12.5 & 10.3 & 9.9 & 9.1 & 23.5 & 9.3 & 16.7 & 0.0 \\
\hline Stap & 7.9 & 8.3 & 6.9 & 6.2 & 6.1 & 11.8 & 7.4 & 0.0 & 0.0 \\
\hline Ener & 7.9 & 8.3 & 10.3 & 12.3 & 12.1 & 0.0 & 7.4 & 16.7 & 33.3 \\
\hline Fin & 21.1 & 20.8 & 34.5 & 7.4 & 27.3 & 33.3 & 37.0 & 33.3 & 66.7 \\
\hline Heal & 7.9 & 8.3 & 13.8 & 11.1 & 6.1 & 9.8 & 11.1 & 16.7 & 0.0 \\
\hline Ind & 31.6 & 25.0 & 0.0 & 21.0 & 15.2 & 3.9 & 16.7 & 0.0 & 0.0 \\
\hline Tech & 13.2 & 16.7 & 13.8 & 24.7 & 18.2 & 17.6 & 11.1 & 0.0 & 0.0 \\
\hline Mat & 5.3 & 0.0 & 6.9 & 4.9 & 6.1 & 0.0 & 0.0 & 16.7 & 0.0 \\
\hline Util & 0.0 & 0.0 & 3.4 & 2.5 & 0.0 & 0.0 & 0.0 & 0.0 & 0.0 \\
\hline
\end{tabular}

Note. The table reports the fraction of Granger and contemporaneous linkages between the industrial sectors. The $(i, j)$ entry of the Granger linkages table is defined as the total number of linkages from sector $j$ to sector $i$ standardized by the total number of linkages from sector $j$. The $(i, j)$ entry of the contemporaneous linkages table is defined as the total number of linkages between sector $i$ and $j$ standardized by the total number of linkages of sector $j$.
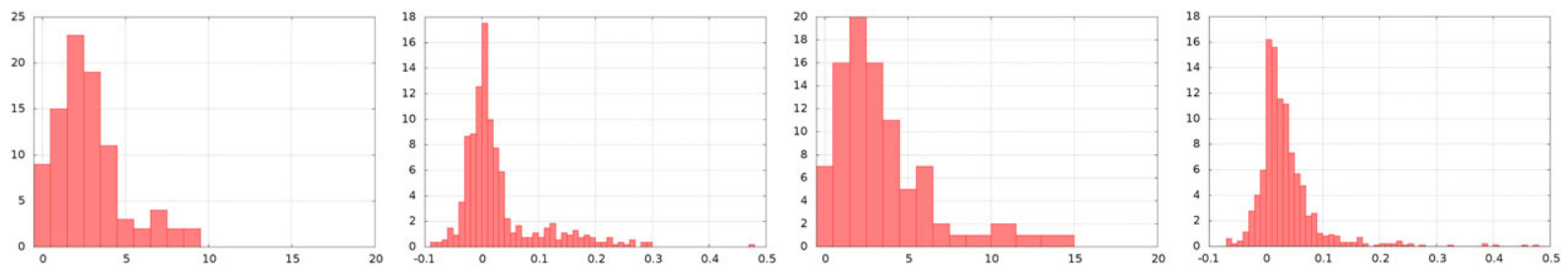

FIGURE 5 Degree and coefficient distributions. The first two pictures from the left display the histogram of the degree distribution of the Granger network and the histogram of the estimated nonzero $\alpha$ coefficients. The last two pictures from the left display the degree distribution of the contemporaneous network and the histogram of the estimated nonzero $\rho$ coefficients [Colour figure can be viewed at wileyonlinelibrary.com]

this criterion in Table 5. The table shows clearly that large financial firms are highly influential. In particular, the results shows that the financial firms that have been heavily involved in the great financial crisis, such as Bank of America (BAC), AIG, and Citigroup (C), are the stocks associated with the largest spillover effects in the Granger network.

Overall, the in-sample estimation results show that, after conditioning on market-wide and sectoral factors, the sparse VAR captures an important proportion of overall variability, and that the financial industry, in particular, has the highest degree of interconnectedness.

Lastly, we investigate the stability of the sparsity patterns of the estimated network through time. In Figure 6 we report the heat map of the adjacency matrices of the Granger and contemporaneous networks estimated from the beginning of the sample until the end of December 2015 (the end of the in-sample period) and until the end of December 2013. We find that the sparsity patterns of the network exhibit a moderate degree of time variation. In particular, we have that for the Granger (contemporaneous) network 80.6\% (85.3\%) of the edges present over the entire sample are also present at the end of December 2013, whereas $98.7 \%(98.1 \%)$ of the edges not present over the entire sample are also not present at the end December 2013. 
TABLE 5 Rankings

\begin{tabular}{lllll} 
& \multicolumn{2}{l}{ Granger } & & \multicolumn{2}{l}{ Contemporaneous } \\
\cline { 2 - 3 } \cline { 5 - 5 } Rank & Company & Sector & Company & Sector \\
\hline 2 & BAC & Financials & UNP & Industrials \\
\hline 3 & AIG & Financials & T & Technology \\
\hline 4 & C & Financials & USB & Financials \\
\hline 5 & MCD & Financials & GE & Industrials \\
\hline 6 & HPQ & Technology & WFC & Financials \\
\hline 7 & DOW & Material & MS & Financials \\
\hline 8 & SPG & Financials & NSC & Industrials \\
9 & GE & Industrials & F & Discretionary \\
\hline 10 & CVS & Staples & NOV & Energy \\
\hline
\end{tabular}

Note. The table reports the top 10 of the most interconnected series in the Granger and contemporaneous networks.
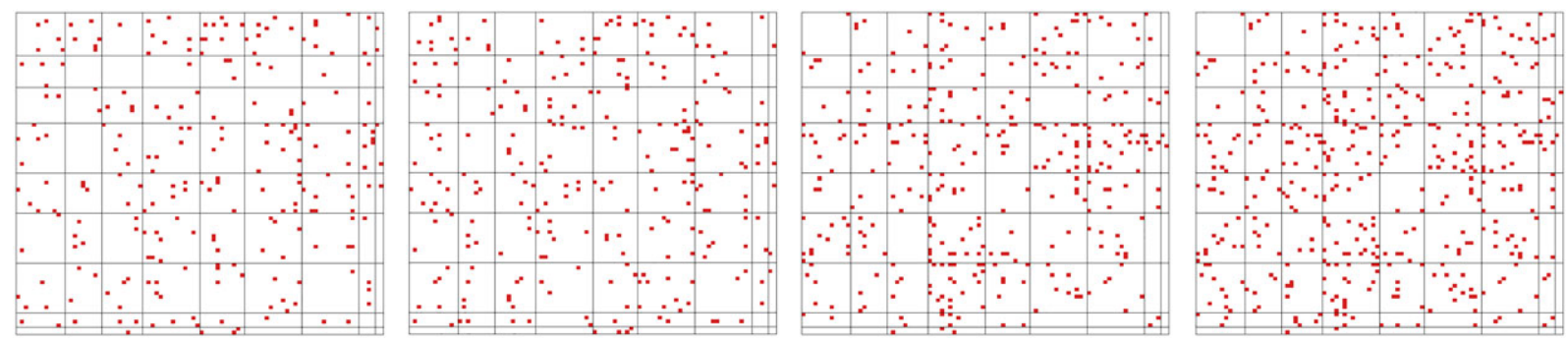

FIGURE 6 Stability of the sparsity patterns. The first two pictures from the left display the adjacency matrix of the Granger network estimated from January 2004 to December 2015 and from January 2004 to December 2013. The last two pictures from the left display the adjacency matrix of the contemporaneous network estimated from January 2004 to December 2015 and from January 2004 to December 2013 [Colour figure can be viewed at wileyonlinelibrary.com]

\section{2 | Out-of-sample forecasting}

We carry out a forecasting exercise to evaluate the out-of-sample performance of the methodology. The exercise is designed as follows. We split the sample into an in-sample period spanning January 2, 2004, to December 31, 2013, and an out-of-sample period spanning January 2, 2014, to December 31, 2015. We first estimate the sparse VAR in-sample using the same steps outlined in the previous section and we then evaluate the model in the out-of-sample period.

The prediction evaluation is divided into two parts. The first part focuses on the evaluation of the nets estimator of the autoregressive component by predicting one-step-ahead volatility residuals. The benchmark forecast for this exercise is the constant zero forecast. Note that the constant zero forecast represents the optimal forecast in case the dependence in the panel is fully captured by the factor part of Model 13 without exploiting the information in the residuals. The competing forecasts are those obtained from a VAR(1) model estimated via net $s$, univariate AR(1) models estimated by least squares, a VAR(1) model estimated by ridge regression and VAR(1) estimated via Bayesian methods. The ridge VAR(1) is fitted by estimating the VAR equation by equation using standard ridge regression. The degree of shrinkage of each equation is chosen via generalized cross-validation. The Bayesian VAR(1) relies on traditional conjugate priors (Koop \& Korobilis, 2009; Sims \& Zha, 1998). Note that the volatility residuals are obtained from the estimation results of Model 13 estimated over the entire sample.

We report the forecasting results in the top panel of Table 6 . The first row of the table reports the MSE of the benchmark, while the remaining rows report the out-of-sample $R^{2}$ of the competitors. The out-of-sample $R^{2}$ index is defined as one minus the ratio of the MSE of the competing models over the MSE of the benchmark. The performance indices are averaged over the entire panel and the industry sectors. The results show that the VAR forecasts obtained by the net s estimator systematically improve forecasting ability over the benchmark by roughly $8 \%$, on average, and it is the best-performing forecast method overall. The ridge and Bayesian VAR do not perform particularly well in this setting 
TABLE 6 Forecasting

\begin{tabular}{lllllllllll} 
& Disc & Stap & Ener & Fin & Heal & Ind & Tech & Mat & Util & All \\
Granger component & & & & & & & & & & \\
benchmark MSE & 5.67 & 3.96 & 4.52 & 3.90 & 5.15 & 4.32 & 5.86 & 8.16 & 3.14 & 4.91 \\
nets $R_{\text {oos }}^{2}$ & 10.21 & 6.25 & 5.30 & 5.86 & 6.98 & 5.46 & 9.49 & 17.90 & 0.50 & 8.08 \\
AR(1) $R_{\text {oos }}^{2}$ & 5.65 & 3.58 & 5.04 & 1.65 & 2.95 & 4.07 & 6.30 & 15.14 & -3.49 & 5.06 \\
ridge $R_{\text {oos }}^{2}$ & 5.47 & 1.79 & 2.74 & -8.46 & 1.09 & 3.39 & 4.89 & 12.84 & -8.20 & 2.57 \\
BVAR $R_{\text {oos }}^{2}$ & 5.89 & 2.19 & 3.53 & -8.20 & 1.36 & 3.77 & 3.47 & 8.70 & -8.78 & 1.63 \\
Contemporaneous component & & & & & & & & & \\
benchmark MSE & 5.12 & 3.74 & 4.30 & 3.66 & 4.82 & 4.11 & 5.35 & 6.78 & 3.11 & 4.54 \\
nets $R_{\text {oos }}^{2}$ & 15.76 & 12.56 & 12.98 & 17.21 & 6.28 & 19.08 & 14.03 & 3.00 & -1.17 & 13.20 \\
reg $R_{\text {oos }}^{2}$ & 12.89 & 9.12 & 9.02 & 15.23 & 2.97 & 17.44 & 10.98 & -1.94 & -6.44 & 10.19 \\
ridge $R_{\text {oos }}^{2}$ & 12.97 & 9.20 & 9.05 & 15.32 & 3.06 & 17.50 & 11.04 & -1.87 & -6.37 & 10.26 \\
\hline
\end{tabular}

Note. The table reports summary forecasting results over the industry sectors and the entire panel. The first panel reports forecasting exercise for the Granger component. The first row reports the MSE $(\times 100)$ of the benchmark. The second to fifth rows report the out-of-sample $R_{\text {oos }}^{2}$ of, respectively, nets, an AR(1) estimated by least squares, a VAR estimated using ridge regression, and a Bayesian VAR. The second panel reports the forecasting exercise of the contemporaneous component. The first row reports the MSE $(\times 100)$ of the benchmark. The second to fourth rows report the out-of-sample $R_{\mathrm{oos}}^{2}$ of, respectively, nets, the linear regression estimator, and the ridge estimator.

but this may be due to the forecasting design. Conditional on the factors the data exhibit a substantially lower degree of collinearity and sparsity appears to be a reasonable modeling assumption. In fact, nets as well as the simple AR(1) end up performing quite well.

The second part focuses on the evaluation of the nets estimator of the contemporaneous component by predicting the contemporaneous volatility residuals conditional on the estimated autoregressive component. We construct the series of VAR residuals $\widehat{\epsilon}_{i t}$ of the autoregressive component estimated by nets, and the focus is on predicting each residual series conditional on the remaining ones on the basis of the regression

$$
\widehat{\epsilon}_{i t}=\sum_{\substack{h=1 \\ h \neq i}}^{n} \gamma_{i j} \widehat{\epsilon}_{h t}+u_{i t}, \quad i=1, \ldots, n .
$$

The benchmark forecast for this exercise is again the constant zero forecast, which is the optimal forecast in case the residuals do not have any cross-correlation. The competing forecasts are those obtained from the contemporaneous component of the VAR estimated by net s, those obtained from a linear regression estimated by least squares, and a linear regression estimated by ridge regression (with tuning parameter chosen by generalized cross-validation). The linear regression and the ridge regression are estimated in the in-sample period using the in-sample one-step-ahead forecast errors.

We report the forecasting results in the bottom panel of Table 6. The first row of the table reports the average MSE of the benchmark model, while the remaining rows report the out-of-sample $R^{2}$ of the competitors. Results show that the nets forecasts systematically improve out-of-sample predictive ability across sectors and, on average, improve forecasting over the benchmark by $13 \%$.

\section{5 | CONCLUSIONS}

In this work we introduce network techniques for the analysis of large panels of time series. We model a panel as a VAR where the autoregressive matrices and the inverse covariance matrix of the system innovations are assumed to be sparse. The system has a natural network representation in terms of a directed graph representing predictive Granger relations and an undirected graph representing contemporaneous partial correlations. A LASSO estimation algorithm called nets is introduced to estimate simultaneously the autoregressive matrices and the inverse covariance matrix of the model. The large-sample properties of the estimator are established in a high-dimensional setting. The methodology is used to analyze a panel of volatility measures of US blue chips between January 2004 and December 2015 conditional on market-wide and sector-specific volatility factors. The analysis shows that the series exhibit a high degree of interconnectedness and that financial firms have the highest degree of interdependence. A forecasting exercise shows that the methodology introduced in this work allows to improve forecasting over a number of benchmarks. 


\section{ACKNOWLEDGMENTS}

Christian Brownlees acknowledges financial support from the Spanish Ministry of Science and Technology (Grant MTM2015-67304-P); from the Spanish Ministry of Economy and Competitiveness, through the Severo Ochoa Programme for Centres of Excellence in R\&D (SEV-2011-0075), and from the Fundación BBVA through the Scientific Research Grant (PR16_DAT_0043) on Analysis of Big Data in Economics and Empirical Applications. We thank the editor Eric Ghysels and two referees for insightful comments which helped improve our paper. We would also like to thank Vasco Carvalho, Frank Diebold, Tony Hall, Nikolaus Hautsch, Julien Idier, Clifford Lam, Marco Lippi, Gabor Lugosi, Miguel Morin, Tommaso Proietti, and Kimmo Soramaki for their helpful comments. Special thanks go to Giorgio Calzolari. All mistakes are ours.

The procedures presented in this paper are available in the package nets for $\mathrm{R}$.

\section{REFERENCES}

Acemoglu, D., Carvalho, V., Ozdaglar, A., \& Tahbaz-Salehi, A. (2012). The network origins of aggregate fluctuations. Econometrica, 80, 1977-2016.

Alizadeh, S., Brandt, M. W., \& Diebold, F. X. (2002). Range-based estimation of stochastic volatility models. Journal of Finance, 57, $1047-1091$.

Andersen, T. G., Bollerslev, T., Diebold, F. X., \& Labys, P. (2003). Modeling and forecasting realized volatility. Econometrica, 71, 579-625.

Ando, T., \& Bai, J. (2017). Clustering huge number of financial time series: A panel data approach with high-dimensional predictors and factor structures. Journal of the American Statistical Association, 112(519), 1182-1198.

Anufriev, M., \& Panchenko, V. (2015). Connecting the dots: Econometric methods for uncovering networks with an application to the Australian financial institutions. Journal of Banking and Finance, 61, S241-S255.

Bai, J. (2003). Inferential theory for factor models of large dimensions. Econometrica, 71, 135-171.

Barigozzi, M., Brownlees, C., Gallo, G. M., \& Veredas, D. (2014). Disentangling systematic and idiosyncratic dynamics in panels of volatility measures. Journal of Econometrics, 182, 364-384.

Barigozzi, M., \& Hallin, M. (2016). Generalized dynamic factor models and volatilities: Recovering the market volatility shocks. Econometrics Journal, 19, C33-C60.

Barigozzi, M., \& Hallin, M. (2017). A network analysis of the volatility of high dimensional financial series. Journal of the Royal Statistical Society, Series C, 66, 581-605.

Basu, S., \& Michailidis, G. (2015). Regularized estimation in sparse high-dimensional time series models. Annals of Statistics, 43, $1535-1567$.

Billio, M., Getmansky, M., Lo, A., \& Pellizzon, L. (2012). Econometric measures of connectedness and systemic risk in the finance and insurance sectors. Journal of Financial Economics, 104, 535-559.

Bosq, D. (1996). Nonparametric statistics for stochastic processes: Estimation and prediction. New York, NY: Springer.

Brownlees, C. T., \& Gallo, G. M. (2010). Comparison of volatility measures: A risk management perspective. Journal of Financial Econometrics, $8,29-56$.

Bühlmann, P. (2013). Statistical significance in high-dimensional linear models. Bernoulli, 19, 1212-1242.

Dahlhaus, R. (2000). Graphical interaction models for multivariate time series. Metrika, 51, 157-172.

Davis, R. A., Zang, P., \& Zheng, T. (2016). Sparse vector autoregressive modeling. Journal of Computational and Graphical Statistics, 25(4), 1077-1096.

De Mol, C., Giannone, D., \& Reichlin, L. (2008). Forecasting using a large number of predictors: Is Bayesian shrinkage a valid alternative to principal components? Journal of Econometrics, 146, 318-328.

Dempster, A. P. (1972). Covariance selection. Biometrics, 28, 157-175.

Den Haan, W. J., \& Levin, A. (1996). Inferences from Parametrics and Non-Parametric Covariance Matrix Estimation Procedures. (NBER Technical Working Paper 195). Cambridge, MA: National Bureau of Economic Research.

Diebold, F. X., \& Yllmaz, K. (2009). Measuring financial asset return and volatility spillovers, with application to global equity markets. Economic Journal, 119, 158-171.

Diebold, F. X., \& Yllmaz, K. (2014). On the network topology of variance decompositions: Measuring the connectedness of financial firms. Journal of Econometrics, 182, 119-134.

Diebold, F. X., \& Yllmaz, K. (2015). Financial and macroeconomic connectedness: A network approach to measurement and monitoring. Oxford, UK: Oxford University Press.

Doukhan, P., \& Neumann, M. H. (2007). Probability and moment inequalities for sums of weakly dependent random variables, with applications. Stochastic Processes and their Applications, 117, 878-903.

Eichler, M. (2007). Granger causality and path diagrams for multivariate time series. Journal of Econometrics, 137, 334-353.

Engle, R. F., Gallo, G. M., \& Velucchi, M. (2012). Volatility spillovers in East Asian financial markets: A MEM-based approach. Review of Economics and Statistics, 94, 222-223.

Fan, J., \& Yao, Q. (2005). Nonlinear time series. New York, NY: Springer.

Foerster, A. T., Sarte, P.-D. G., \& Watson, M. W. (2011). Sectoral versus aggregate shocks: A structural factor analysis of industrial production. Journal of Political Economy, 119(1), 1-38. 
Forni, M., Hallin, M., Lippi, M., \& Reichlin, L. (2000). The generalized dynamic factor model: Identification and estimation. Review of Economics and Statistics, 82, 540-554.

Friedman, J., Hastie, T., \& Tibshirani, R. (2008). Sparse inverse covariance estimation with the graphical lasso. Biostatistics, 9, 432-441.

Fu, W. J. (1998). Penalized regression: The bridge versus the lasso. Journal of Computational and Graphical Statistics, 7, $397-416$.

Giannone, D., Lenza, M., \& Primicieri, G. E. (2018). Economic predictions with big data: The illusion of sparsity. (Staff Reports 847). New York, NY: Federal Reserve Bank.

Hagströmer, B., \& Menkveld, A. J. (2016). A network map of information percolation. (Technical Report). Stockholm Sweden/Amsterdam, Netherlands: Stockholm Business School and VU University Amsterdam.

Härdle, W. K., Wang, W., \& Yu, L. (2016). TENET: Tail-Event driven network risk. Journal of Econometrics, 192(2), 499-513.

Hautsch, N., Schaumburg, J., \& Schienle, M. (2015). Financial network systemic risk contributions. Review of Finance, 19(2), 685-738.

Huang, J., Ma, S., \& Zhang, C.-H. (2008). Adaptive lasso for sparse high-dimensional regression models. Statistica Sinica, $18,1603-1618$.

Kock, A. B. (2016). Consistent and conservative model selection with the adaptive lasso in stationary and nonstationary autoregressions. Econometric Theory, 32, 243-259.

Kock, A. B., \& Callot, L. (2015). Oracle inequalities for high dimensional vector autoregressions. Journal of Econometrics, 186, 325-344.

Koop, G., \& Korobilis, D. (2009). Bayesian multivariate time series methods for empirical macroeconomics. Foundations and Trends in Econometrics, 3, 267-358.

Lauritzen, S. L. (1996). Graphical models. Oxford, UK: Clarendon Press.

Ledoit, O., \& Wolf, M. (2004). A well-conditioned estimator for large-dimensional covariance matrices. Journal of Multivariate Analysis, 88, 365-411.

Mantegna, R. (1999). Hierarchical structure in financial markets. European Physical Journal B, 11, 193-197.

Medeiros, M. C., \& Mendes, E. F. (2016). 11-regularization of high-dimensional time-series models with non-Gaussian and heteroskedastic errors. Journal of Econometrics, 191, 255-271.

Meinshausen, N., \& Bühlmann, P. (2006). High dimensional graphs and variable selection with the lasso. Annals of Statistics, 34, 1436-1462.

Merlevède, F., Peligrad, M., \& Rio, E. (2011). A Bernstein type inequality and moderate deviations for weakly dependent sequences. Probability Theory and Related Fields, 151, 435-474.

Onatski, A. (2012). Asymptotics of the principal components estimator of large factor models with weakly influential factors. Journal of Econometrics, 168(2), 244-258.

Parkinson, M. (1980). The extreme value method for estimating the variance of the rate of return. Journal of Business, 53, 61-65.

Peng, J., Wang, P., Zhou, N., \& Zhu, J. (2009). Partial correlation estimation by joint sparse regression models. Journal of the American Statistical Association, 104, 735-746.

Shao, J., \& Deng, X. (2012). Estimation in high-dimensional linear models with deterministic design matrices. Annals of Statistics, 40, 812-831.

Sims, C. A., \& Zha, T. (1998). Bayesian methods for dynamic multivariate models. International Economic Review, 39, 949-968.

Song, S., \& Bickel, P. (2011). Large vector auto regressions. (SFB 649 Discussion Paper 2011-048). Berlin, Germany: Humboldt University.

Stock, J. H., \& Watson, M. W. (2002). Forecasting using principal components from a large number of predictors. Journal of the American Statistical Association, 97, 1167-1179.

Wang, F., \& Ghysels, E. (2015). Econometric analysis of volatility component models. Econometric Theory, 31(2), $362-393$.

Zou, H. (2006). The adaptive lasso and its oracle properties. Journal of the American Statistical Association, 101, 1418-1429.

\section{SUPPORTING INFORMATION}

Additional supporting information may be found online in the Supporting Information section at the end of the article.

How to cite this article: Barigozzi M, Brownlees C. Nets: Network estimation for time series. J Appl Econ. 2019;34:347-364. https://doi.org/10.1002/jae.2676 